\title{
Inverse Scattering Problem for the Schrödinger Equation with Magnetic Potential at a Fixed Energy
}

\author{
G. Eskin, J. Ralston
}

Department of Mathematics, University of California, Los Angeles, CA 90024, USA

Received: 1 August 1994/in revised form: 3 January 1995

\begin{abstract}
In this article we consider the Schrödinger operator in $R^{n}, n \geqq 3$, with electric and magnetic potentials which decay exponentially as $|x| \rightarrow \infty$. We show that the scattering amplitude at fixed positive energy determines the electric potential and the magnetic field.
\end{abstract}

\section{Introduction}

Consider the Schrödinger equation in $R^{n}, n \geqq 3$, with magnetic potential $A(x)=$ $\left(A_{1}(x), \ldots, A_{n}(x)\right)$ and electric potential $V(x)$ :

$$
-\sum_{j=1}^{n}\left(\frac{\partial}{\partial x_{j}}+i A_{j}(x)\right)^{2} u+V(x) u=k^{2} u
$$

$k>0$, or equivalently

$$
-\Delta u-2 i \sum_{j=1}^{n} A_{j}(x) \frac{\partial u}{\partial x_{j}}+q(x) u=k^{2} u
$$

where

$$
q(x)=\sum_{j=1}^{n}\left(A_{j}^{2}(x)-i \frac{\partial A_{j}}{\partial x_{j}}\right)+V(x) .
$$

We will assume that the potentials $A$ and $V$ are real-valued and exponentially decreasing, i.e.

$$
\left|\frac{\partial^{\alpha} V(x)}{\partial x^{\alpha}}\right| \leqq C_{\alpha} e^{-\delta|x|}, \quad\left|\frac{\partial^{\beta} A_{j}}{\partial x^{\beta}}\right| \leqq C_{\beta} e^{-\delta|x|}, \quad j=1, \ldots, n,
$$

for $0 \leqq|\alpha| \leqq P, 0 \leqq|\beta| \leqq P+1$, where $P=n+4$. We consider the solutions of (1) of the form

$$
u=e^{i k(1) \cdot x}+v(x, \omega, k) \text {, }
$$

${ }^{*}$ This research was supported by National Science Foundation Grant DMS93-05882. 
where $v$ is the outgoing solution of

$$
-\Delta v-2 i \sum_{j=1}^{n} A_{j}(x) \frac{\partial v}{\partial x_{j}}+\left(q(x)-k^{2}\right) v=e^{i k \omega} \cdot x\left(-2 k \sum_{j=1}^{n} \omega_{j} A_{j}(x)-q(x)\right)
$$

obtained by the limiting absorption method. By this argument $v$ exists and is unique whenever $k^{2}$ is not an embedded eigenvalue, and, combining Sect. 5 of Hörmander [4] with the proof of Theorem 3.3 of Agmon [1], one sees that (3) implies there are no embedded eigenvalues. Representing $v$ in terms of the outgoing fundamental solution of $\Delta+k^{2}$, it follows that as $|x| \rightarrow \infty$,

$$
v(x, \omega, k)=\frac{e^{\imath k|x|}}{|x|^{\frac{n-1}{2}}}\left(a\left(\frac{x}{|x|}, \omega, k\right)+O\left(\frac{1}{|x|}\right)\right),
$$

where $a(\theta, \omega, k)$ is defined to be the scattering amplitude. Our objective is to prove

Theorem 1. Fix $k>0$. Then one can recover $V(x)$ and the magnetic field $B=$ curl $A$ from the scattering amplitude $a(\theta, \omega, k),(\theta, \omega) \in S^{n-1} \times S^{n-1}$.

Note that, if $A$ and $A^{\prime}$ satisfy (3) and curl $A=\operatorname{curl} A^{\prime}$, then $A^{\prime}-A$ is the gradient of function $\varphi$ satisfying

$$
\left|\frac{\partial^{p} \varphi}{\partial x^{p}}\right| \leqq C_{p} e^{-\delta|x|}, \quad 0 \leqq|p| \leqq P
$$

To see that changing $A$ to $A^{\prime}=A+\frac{\partial \varphi}{\partial x}$ does not change the scattering amplitude note that, if one replaces $u(x)$ by $w(x)=u(x) e^{-l \varphi(x)}$, then $w(x)$ will satisfy

$$
-\left(\frac{\partial}{\partial x}+i A(x)+i \frac{\partial \varphi}{\partial x}\right)^{2} w+V(x) w=k^{2} w .
$$

However, this does not change the scattering amplitude, since

$$
\begin{aligned}
w & =u(x) e^{-i \varphi(x)}=e^{-i \varphi(x)}\left(e^{i k \omega \cdot x}+a\left(\frac{x}{|x|}, \omega, k\right) \frac{e^{i k|x|}}{|x|^{\frac{n-1}{2}}}+O\left(\frac{1}{|x|^{\frac{n+1}{2}}}\right)\right) \\
& =e^{i k \omega \cdot x}+a\left(\frac{x}{|x|}, \omega, k\right) \frac{e^{i k|x|}}{|x|^{\frac{n-1}{2}}}+O\left(\frac{1}{|x|^{\frac{n+1}{2}}}\right) .
\end{aligned}
$$

In this article as in [2] we will use $h(\xi, k \omega, k)$, the Fourier transform of $-\left(\Delta+k^{2}\right) v$, to study the scattering amplitude. Since $v$ is obtained by limiting absorption,

$$
v(x, \omega, k)=\frac{1}{(2 \pi)^{n}} \int_{\mathbb{R}^{n}} \frac{h(\xi, k \omega, k) e^{i x \cdot \xi}}{|\xi|^{2}-k^{2}-i 0} d \xi,
$$

and, taking the asymptotics of (8) when $\theta=x /|x|$ is fixed and $|x| \rightarrow \infty$, one obtains

$$
a(\theta, \omega, k)=C_{n, k} h(k \theta, k \omega, k), C_{n, k}=\frac{1}{4 \pi}\left(\left(\frac{k}{2 \pi}\right)^{\frac{1}{2}} e^{-\frac{i \pi}{4}}\right)^{n-3} .
$$


From (5) one sees that $h$ satisfies

$$
h(\xi, \zeta, k)+\frac{1}{(2 \pi)^{n}} \int_{\mathbb{R}^{n}} \frac{q_{0}(\xi-\eta, \eta) h(\eta, \zeta, k)}{|\eta|^{2}-k^{2}-i 0} d \eta=-q_{0}(\xi-\zeta, \zeta),
$$

where

$$
q_{0}(\xi, \zeta)=2 \sum_{j=1}^{n} \hat{A}_{j}(\xi) \zeta_{j}+\hat{q}(\xi)
$$

Note that (3) implies that $q_{0}(\xi-\zeta, \zeta)$ is analytic in $(\xi, \zeta)$ for $|\operatorname{Im} \xi|<\delta / 2$, $|\operatorname{Im} \zeta|<\delta / 2$. For fixed $\lambda$, the integral operator

$$
T_{\lambda} w=\frac{1}{(2 \pi)^{n}} \int_{\mathbb{R}^{n}} \frac{q_{0}(\xi-\eta, \eta) w(\eta)}{|\eta|^{2}-\lambda-i 0} d \eta
$$

is compact in the space $H_{\alpha, N}, 0<\alpha<1, n-1<N<n+4$. Here $H_{\alpha, N}$ is the weighted Hölder space used in [2]: let $\|f\|_{\gamma, N}=\left\|\left(1+|\xi|^{2}\right)^{N / 2} f\right\|_{\alpha}$, where \|\|$_{\alpha}$. is the standard Hölder norm, and define $H_{\alpha, N}$ as the completion of $C_{0}^{\infty}\left(R^{n}\right)$ is \|\|$_{\alpha, N}$. Moreover, $T_{\lambda}$ depends analytically on $\lambda$ for $\operatorname{Im} \lambda>0$ and extends continuously to the positive real axis, $\lambda>0$. In the same way that Theorem 5.2 of [4] showed that the homogeneous equation corresponding to (5) had no nontrivial square-integrable solutions, it can be used here to show the $I+T_{k^{2}}$ has no nontrivial solutions in $H_{\alpha, N}\left(R^{n}\right)$. Hence we see that the Fredholm operator $I+T_{k^{2}}$ is invertible on $H_{\alpha, N}$ for $k>0$. This will be useful in what follows.

In the case that the magnetic field $B$ is small uniqueness results at fixed energy have been obtained previously by Henkin and Novikov [6] and by Sun [9]. Recently Nakamura, Sun and Uhlmann [5] obtained the uniqueness result analogous to Theorem 1 for the Dirichlet to Neumann map. This implies Theorem 1 for magnetic and electric potentials of compact support. In fact, when the magnetic and electric potentials have compact support, as in [9], uniqueness for inverse scattering at fixed energy and uniqueness for the Dirichlet-to-Neumann map inverse problem at fixed energy are equivalent.

For potentials without compact support the previous work which influenced us considerably was by Novikov [8]. He proved Theorem 1 in the case of zero magnetic potential, and the methods of [8] could be used to give a different proof of some of the results in Sect. 2.

Finally, we are deeply indebted to Adrian Nachman for calling our attention to a serious error in the first version of Sect. 2 .

\section{Faddeev-Type Scattering Amplitudes}

Following Faddeev [3] and Novikov-Khenkin [6], we introduce a new scattering amplitude which will contain a large parameter. The later will be helpful in solving the inverse scattering problem.

Let $v$ be an arbitrary unit vector, $|v|=1$, and $E_{v, \sigma}(x)$ be the following fundamental solution to the equation $\left(-\Delta-k^{2}\right) u=f$ :

$$
E_{v, \sigma}(x)=\frac{1}{(2 \pi)^{n}} \int_{\mathbb{R}^{n}} \frac{e^{i x} \cdot \eta d \eta}{\eta \cdot \eta-k^{2}+i 0\left(\eta^{\prime}-\sigma\right)},
$$


where $\eta_{v}=\eta \cdot v$ and $-k<\sigma<k$. Comparing $E_{v, \sigma}(x)$ with the fundamental solution

$$
E_{0}(x)=\frac{1}{(2 \pi)^{n}} \int_{\mathbb{R}^{n}} \frac{e^{i x \cdot \eta}}{\eta \cdot \eta-k^{2}-i 0} d \eta,
$$

we have

$$
E_{v, \sigma}(x)=E_{0}(x)-\frac{i \pi k^{n-2}}{(2 \pi)^{n}} \int_{k(1) \cdot v>\sigma} e^{l x \cdot k \omega} d \omega,
$$

where $d \omega$ is the area element of the unit sphere in $R^{n}$. Analogously to (10) consider the following integral equation

$$
h_{v, \sigma}(\xi, \zeta, k)+\frac{1}{(2 \pi)^{n}} \int_{\mathbb{R}^{n}} \frac{q_{0}(\xi-\eta, \eta) h_{v, \sigma}(\eta, \zeta, k)}{\eta \cdot \eta-k^{2}+i 0\left(\eta_{v}-\sigma\right)} d \eta=-q_{0}(\xi-\zeta, \zeta) .
$$

Set

$$
v_{v, \sigma}(x, \zeta, k)=\frac{1}{(2 \pi)^{n}} \int_{\mathbb{R}^{n}} \frac{h_{v, \sigma}(\xi, \zeta, k) e^{\imath x \cdot \xi}}{\xi \cdot \xi-k^{2}+i 0\left(\xi_{v}-\sigma\right)} d \xi,
$$

assuming that $h_{v, \sigma}(\xi, \zeta, k)$ is the solution of (16). Then $v_{v, \sigma}(x, \zeta, k)$ is a solution of the differential equation (5) for $\zeta=k \omega$ with asymptotics at infinity that can be obtained by applying the stationary phase method to (17).

Now we shall find the relation between $h_{v, \sigma}(\xi, \zeta, k)$ and $h(\xi, \zeta, k)$. Analogously to (15) we have

$$
\begin{aligned}
& \frac{1}{(2 \pi)^{n}} \int_{\mathbb{R}^{n}} \frac{q_{0}(\xi-\eta, \eta) h_{v, \sigma}(\eta, \zeta, k)}{\eta \cdot \eta-k^{2}+i 0\left(\eta_{v}-\sigma\right)} d \eta=\frac{1}{(2 \pi)^{n}} \int_{\mathbb{R}^{n}} \frac{q_{0}(\xi-\eta, \eta) h_{v, \sigma}(\eta, \zeta, k)}{\eta \cdot \eta+k^{2}-i 0} d \eta \\
& \quad-\frac{i \pi k^{n-2}}{(2 \pi)^{n}} \int_{k \omega} q_{v>\sigma}(\xi-k \omega, k \omega) h_{v, \sigma}(k \omega, \zeta, k) d \omega .
\end{aligned}
$$

It follows from (16) and (18) that

$$
\begin{aligned}
& h_{v, \sigma}(\xi, \zeta, k)+\frac{1}{(2 \pi)^{n}} \int_{\mathbb{R}^{n}} \frac{q_{0}(\xi-\eta, \eta) h_{v, \sigma}(\eta, \zeta, k)}{\eta \cdot \eta-k^{2}-i 0} d \eta \\
& \quad=-\frac{i \pi k^{n-2}}{(2 \pi)^{n}} \int_{k \omega} q_{v>\sigma}(\xi-k \omega, k \omega) h_{v, \sigma}(k \omega, \zeta, k) d \omega-q_{0}(\xi-\zeta, \zeta) .
\end{aligned}
$$

Set

$$
A\left(q_{0}\right) w=\frac{1}{(2 \pi)^{n}} \int_{\mathbb{R}^{n}} \frac{q_{0}(\xi-\eta, \eta) w(\eta)}{\eta \cdot \eta-k^{2}-i 0} d \eta
$$

and

$$
A(h) w=\frac{1}{(2 \pi)^{n}} \int_{\mathbb{R}^{n}} \frac{h(\xi, \eta, k) w(\eta)}{\eta \cdot \eta-k^{2}-i 0} d \eta .
$$

That (10) has a unique solution is equivalent (cf. [2]) to the equality

$$
\left(I+A\left(q_{0}\right)\right)(I+A(h))=I .
$$


Since $I+A\left(q_{0}\right)$ has an inverse, it follows from (22) that

$$
(I+A(h))\left(I+A\left(q_{0}\right)\right)=I
$$

or equivalently

$$
h(\xi, \zeta, k)+q_{0}(\xi-\zeta, \zeta)+\frac{1}{(2 \pi)^{n}} \int_{\mathbb{R}^{n}} \frac{h(\xi, \eta, k) q_{0}(\eta-\zeta, \zeta)}{\eta \cdot \eta-k^{2}-i 0} d \eta=0 .
$$

Applying $I+A(h)$ to (19) and using (23) and $\left(23^{\prime}\right)$, we obtain (cf. [3] and [6], formula (1.7)):

$$
h_{v, \sigma}(\xi, \zeta, k)=h(\zeta, \zeta, k)-\frac{i \pi k^{n-2}}{(2 \pi)^{n}} \int_{v \omega} h(\xi, k \omega, k) h_{v, \sigma}(k \omega, \zeta, k) d \omega .
$$

Since $I+A\left(q_{0}\right)$ is invertible, Eq. (24) has a unique solution for any $h(\xi, \zeta, k)$ if and only if Eq. (16) has a unique solution. Indeed, if $\varphi(\xi)$ is a solution of the homogeneous equation corresponding to (16), i.e.

$$
\varphi(\xi)+(2 \pi)^{-n} \int_{\mathbb{R}^{n}} \frac{q_{0}(\xi-\eta, \eta) \varphi(\eta)}{\eta \cdot \eta-k^{2}+i 0\left(\eta_{v}-\sigma\right)} d \eta=0
$$

then from (25) and (18) with $h_{v}$ replaced with $\varphi$ we conclude that

$$
\varphi(\xi)+(2 \pi)^{-n} \int_{\mathbb{R}^{n}} \frac{q_{0}(\xi-\eta, \eta) \varphi(\eta) d \eta}{\eta \cdot \eta-k^{2}-i 0}=\frac{i \pi k^{n-2}}{(2 \pi)^{n}} \int_{k \omega} q_{v>\sigma}(\xi-k \omega, k \omega) \varphi(k \omega) d \omega
$$

Applying $(I+A(h))$ to both sides of this, we have

$$
0=\varphi(\xi)+\frac{i \pi k^{n-2}}{(2 \pi)^{n}} \int_{v \omega} h(\xi, k \omega, k) \varphi(k \omega) d \omega
$$

i.e. $\varphi$ restricted to $|\xi|=k$ solves the homogeneous equation corresponding to (24). Conversely, suppose $\varphi(\xi)$ is a nonzero solution of the preceding equation (26) on the sphere of radius $k$. Then (26) extends $\varphi$ to $R^{n}$, since $h(\xi, k \omega, k)$ is defined for $\xi \in R^{n}$. Applying $I+A\left(q_{0}\right)$ to both sides of (26), we see that $\varphi$ satisfies (25).

Denote by $E_{1}(x, z)$ the following function:

$$
E_{v}(x, z)=\frac{1}{(2 \pi)^{n}} \int_{\mathbb{R}^{n}} \frac{e^{i x} \cdot \eta d \eta}{(\eta+z v) \cdot(\eta+z v)-k^{2}}, \quad \operatorname{Im} z>0 .
$$

Note that $E_{v}(x, z)$ is a fundamental solution for $\left(-i \frac{\partial}{\partial x}+z v\right) \cdot\left(-i \frac{\partial}{\partial x}+z v\right)-k^{2}$, i.e.

$$
\left[\left(-i \frac{\partial}{\partial x}+z v\right) \cdot\left(-i \frac{\partial}{\partial x}+z v\right)-k^{2}\right] E_{v}(x, z)=\delta(x) .
$$

Note that the distribution $\left[(\eta+z v) \cdot(\eta+z v)-k^{2}\right]^{-1}$ is not analytically dependent on $z$ for $\operatorname{Im} z>0$. This gives rise to the $\bar{\partial}$-equation in inverse scattering (see, for example [6]). 
Denote by $h_{v}(\xi, \zeta, k, z)$ the solution of the following integral equation:

$$
\begin{aligned}
& h_{v}(\xi, \zeta, k, z)+\frac{1}{(2 \pi)^{n}} \int_{\mathbb{R}^{n}} \frac{q_{0}(\xi-\eta, \eta+z v) h_{v}(v, \zeta, k, z)}{(\eta+z v) \cdot(\eta+z v)-k^{2}} d \eta \\
& \quad=-q_{0}(\xi-\zeta, \zeta+z v), \quad z=i \tau, \quad \tau>0 .
\end{aligned}
$$

Let $T_{l \tau}^{(1)}$ denote the operator

$$
\left[T_{l \tau}^{(1)} f\right](\xi)=(2 \pi)^{-n} \int_{\mathbb{R}^{n}} \frac{q_{0}(\xi-\eta, \eta+i \tau \nu) f(\eta) d \eta}{(\eta+i \tau \nu) \cdot(\eta+i \tau \nu)-k^{2}} .
$$

Then (27) can be written

$$
\left[\left(I+T_{\imath \tau}^{(1)}\right) h_{v}\right](\xi)=-q_{0}(\xi-\zeta, \zeta+i \tau v)
$$

and

$$
h_{v}(\xi, \zeta, k, i \tau)=-\left[\left(I+T_{i \tau}\right)^{-1} q_{0}(\cdot-\zeta, \zeta+i \tau v)\right](\xi),
$$

provided $\left(I+T_{i \tau}^{(1)}\right)^{-1}$ exists. The analyticity of $h_{v}$ in $\tau$ will be important for us. Thus we need to study the analyticity of $T_{i \tau}^{(1)} f$ in $\tau$ when $f(\eta)$ is analytic in a strip $|\operatorname{Im} \eta|<\varepsilon$. We will use coordinates $\eta_{v}=\eta \cdot v, \quad \eta^{\prime}=\eta-\eta_{v} v, \quad r=\left|\eta^{\prime}\right| \quad$ and $\omega^{\prime}=\eta^{\prime} /\left|\eta^{\prime}\right|$. For $\eta$ real and $\tau=\mu+i \sigma$,

$$
\operatorname{Im}\left((\eta+i \tau v) \cdot(\eta+i \tau v)-k^{2}\right)=2 \mu \eta_{v}-2 \mu \sigma .
$$

Hence, for $\left|\eta_{v}\right|>\varepsilon_{1}$, Re $\tau>0$ and $|\operatorname{Im} \tau|<\varepsilon_{1} / 2$ the denominator in the integral defining $T_{i \tau}^{(1)}$ does not vanish. Thus, choosing $\chi \in C_{0}^{\infty}(R)$ such that $\chi(s)$ is supported in $|s|<2 \varepsilon_{1}$ and $1-\chi(s)$ is supported in $|s|>\varepsilon_{1}$, we have

$$
\begin{aligned}
{\left[T_{\imath \tau}^{(1)} f\right](\xi)=} & (2 \pi)^{-n} \int_{\mathbb{R}^{n}} \frac{\chi\left(\eta_{v}\right) q_{0}(\xi-\eta, \eta+i \tau v) f(\eta) d \eta}{(\eta+i \tau v) \cdot(\eta+i \tau v)-k^{2}} \\
& +(2 \pi)^{-n} \int_{\mathbb{R}^{n}} \frac{\left(1-\chi\left(\eta_{v}\right)\right) q_{0}(\xi-\eta, \eta+i \tau v) f(\eta) d \eta}{(\eta+i \tau v) \cdot(\eta+i \tau v)-k^{2}} \\
\equiv & {\left[V_{\tau}^{(1)} f\right](\xi)+\left[V_{\tau}^{(2)} f\right](\xi), }
\end{aligned}
$$

where $\left[V_{\tau}^{(2)} f\right](\xi)$ is analytic in $(\xi, \tau)$ in the set $|\operatorname{Im} \xi|<\delta, \operatorname{Re} \tau>0$ and $|\operatorname{Im} \tau|<\varepsilon_{1} / 2$.

In our coordinates we have

$$
(\eta+i \tau v) \cdot(\eta+i \tau v)-k^{2}=(r-\sqrt{B})(r+\sqrt{B}),
$$

where $B=k^{2}+\left(\tau-i \eta_{v}\right)^{2}$. Using $\tau=\mu+i \sigma$ again, we have $\operatorname{Re} B=k^{2}+\mu^{2}-$ $\sigma^{2}+2 \sigma \eta_{v}-\eta_{v}^{2}$, and $\operatorname{Im} B=2 \mu \sigma-2 \mu \eta_{v}$. Hence for $k^{2}>8 \varepsilon_{1}^{2}, \operatorname{Re} B>k^{2} / 8$ for $\left|\eta_{v}\right|<2 \varepsilon_{1}$ and $|\operatorname{Im} \tau|<\varepsilon_{1} / 2$, and we fix $\sqrt{B}$ as the square root in the right half plane. We wish to define $V_{\tau}^{(1)}$, and hence $T_{\imath \tau}^{(1)}$, by analytic continuation from $\tau>0$. When $\tau>0$, i.e. when $\mu>0$ and $\sigma=0, r-\sqrt{B} \neq 0$ for $\eta_{v} \neq 0$, and we have sgn $(\operatorname{Im} B)=-\operatorname{sgn} \eta_{v}$. Therefore, we will deform the integration in $r$ in

$$
\left[V_{\tau}^{(1)} f\right](\xi)=\int_{S^{n-2}} d \omega^{\prime} \int_{\mathbb{R}} d \eta_{v}\left(\int_{0}^{\infty} \frac{\chi\left(\eta_{v}\right) q_{0}(\xi-\eta, \eta+i \tau v) f(\eta) r^{n-2}}{(r-\sqrt{B})(r+\sqrt{B})} d r\right)
$$


into the upper half plane for $\eta_{v}>0$ and into the lower half plane for $\eta_{v}<0$. We need to deform $[0, \infty)$ far enough that $r-\sqrt{B}$ will not vanish on the new contour for $\tau$ in a complex neighborhood of $\left[0, \tau_{0}\right]$. Note that for $\tau=\mu+i \sigma$,

$$
\begin{aligned}
\sqrt{B} & =\sqrt{\mu^{2}+k^{2}+2 i\left(\sigma-\eta_{v}\right) \mu-\left(\sigma-\eta_{v}\right)^{2}} \\
& =\sqrt{\mu^{2}+k^{2}}+i\left(\sigma-\eta_{v}\right) \frac{\mu}{\sqrt{\mu^{2}+k^{2}}}+O\left(\left(\sigma-\eta_{v}\right)^{2}\right) .
\end{aligned}
$$

Hence, for $|\sigma|<\varepsilon_{1} / 2$ and $\left|\eta_{v}\right|<2 \varepsilon_{1}$, we have $\left|\operatorname{Re}\left(\sqrt{B}-\sqrt{\mu^{2}+k^{2}}\right)\right|<C \varepsilon_{1}^{2}$ and $|\operatorname{Im} \sqrt{B}|<5 \varepsilon_{1} / 2+C \varepsilon_{1}^{2}$. We now fix $\varepsilon_{1}>0$ such that $C \varepsilon_{1}^{2}<k / 3,5 \varepsilon_{1} / 2+C \varepsilon_{1}^{2}<$ $\varepsilon / 2$ and $8 \varepsilon_{1}^{2}<k^{2}$. Then we may deform the $r$ integration in $V_{t}^{(1)} f$ to the piecewise linear curve $\Gamma$ from 0 to $k / 2$ to $k / 2+i \varepsilon / 2 \operatorname{sgn} \eta_{v}$ to $\sqrt{k^{2}+\tau_{0}^{2}}+k / 2+i \varepsilon / 2$ sgn $\eta_{v}$ to $\sqrt{k^{2}+\tau_{0}}+k / 2$ to $\infty$. With this choice of $\Gamma, r-\sqrt{B}$ will not vanish on $\Gamma$ for $\left|\eta_{v}\right|<2 \varepsilon_{1},|\sigma|<\varepsilon_{1} / 2$ and $0 \leqq \mu \leqq \tau_{0}$. Thus we have proven:

Lemma 1. If $f(\eta)$ is analytic in $|\operatorname{Im} \eta|<\varepsilon$, satisfying $|f(\eta)| \leqq C(1+|\eta|)^{-n-1}$ for $|\operatorname{Im} \eta|<\varepsilon$, then $\left[T_{i \tau}^{(1)} f\right](\xi)$ has an analytic extension from $\tau>0$ to the half strip $\left\{(\xi, \tau):|\operatorname{Im} \xi|<\delta-\varepsilon\right.$, $\left.\operatorname{Re} \tau>0,|\operatorname{Im} \tau|<\varepsilon_{1} / 2\right\}$.

Let $A_{N, r}$ denote the space of functions $f(\eta)$, analytic on $S_{r}=\left\{\eta \in C^{n}\right.$ : $|\operatorname{Im} \eta|<r\}$ and continuous on $\bar{S}_{r}$, which satisfy

$$
|f(\eta)| \leqq C(1+|\eta|)^{-N}
$$

on $S_{r} . A_{N, r}$ is a Banach space in the norm

$$
\|f\|_{N, r}=\sup _{S_{1}}(1+|\eta|)^{N}|f(\eta)|
$$

Proposition 1. For $\varepsilon_{1}$ sufficiently small $T_{i \tau}^{(1)}$ is a family of compact operators on $A_{n+1, \delta / 3}$, depending continuously on $\tau$ in the closed half strip $D=\{\tau=\mu+i \sigma$ : $\left.\mu \geqq 0,|\sigma| \leqq \varepsilon_{1} / 2\right\}$ and analytically on $\tau$ in $\stackrel{\circ}{D}$, the interior of $D$.

Remark 1. The choice $N=n+1$ is made simply to make the Banach spaces used here compatible with those used in Sect. 3. The $\delta$ here is from (3).

Proof. For $\tau \in \stackrel{\circ}{D}, T_{l \tau}^{(1)} f=V_{\tau}^{(1)} f+V_{\tau}^{(2)} f$ by definition. Since $r^{2}+\left(\eta_{v}+i \tau\right)^{2}-k^{2}$ does not vanish for $r \in \Gamma$ and $\tau \in D$, the operator $V_{\tau}^{(1)}$ satisfies

$$
\left|\left[V_{\tau}^{(1)} f\right](\xi)\right| \leqq C_{\tau} \int_{S^{n-2}} d \omega^{\prime} \int_{\mathbb{R}} d \eta_{v} \int_{\Gamma} \frac{\left|q_{0}(\xi-\eta, \eta+i \tau)\|f(\eta)\| r^{n-2} \| d r\right|}{(1+|\eta|)^{2}}
$$

where the constant $C_{\tau}$ is uniformly bounded on compact subsets of $D$. By hypothesis (3) for any $\delta^{\prime}<\delta$,

$$
\left|q_{0}(\xi-\eta, \eta+i \tau v)\right| \leqq C_{\tau, \delta^{\prime}}(1+|\xi-\eta|)^{-n-4}(1+|\eta|)
$$


for $\xi \in S_{\delta^{\prime}-\varepsilon}$ and $\eta \in S_{\varepsilon}$, where again $C_{\tau, \delta^{\prime}}$ is uniformly bounded on compact subsets of $D$. Since $|f(\eta)| \leqq(1+|\eta|)^{-n-1}\|f\|_{n+1, \varepsilon}$ on $S_{\varepsilon}$, the integrand in (29) is bounded by

$$
C_{\tau, \delta^{\prime}} \frac{\left|r^{n-2}\right|}{(1+|\xi-\eta|)^{n+2}(1+|\eta|)^{n+2}} .
$$

Since for any $p>0$,

$$
(1+|\xi|)^{p}(1+|\xi-\eta|)^{-p}(1+|\eta|)^{-p} \leqq C\left((1+|\xi-\eta|)^{-p}+(1+|\eta|)^{-p}\right),
$$

we conclude

$$
(1+|\xi|)^{n+2}\left|\left[V_{\tau}^{(1)} f\right](\xi)\right| \leqq C\|f\|_{n+1, \varepsilon} .
$$

Taking $\varepsilon=\delta / 3$ and $\delta^{\prime}=5 \delta / 6$, we have $\left[V_{\tau}^{(1)} f\right](\xi)$ analytic in $S_{\delta / 2}$. Thus for $\tau \in D$, $V_{\tau}^{(1)}$ maps $A_{n+1, \delta / 3}$ into $A_{n+2, \delta / 2}$ with norm uniformly bounded on compact subsets of $D$. Hence $V_{\tau}^{(1)}$ is compact for $\tau \in D$.

In proving Lemma 1 we showed that for $f \in A_{n+1, \delta / 3},\left[V_{\tau}^{(1)} f\right](\xi)$ was analytic in $(\xi, \tau)$ for $\tau \in D$ and $\xi \in S_{\delta / 2}$. Since the norm of $V_{\tau}^{(1)}$ as an operator on $A_{n+1, \delta / 3}$ is uniformly bounded on compact subsets it follows by Cauchy's formula that $V_{\tau}^{(1)}$ is an analytic family of operators for $\tau \in D$.

For $\tau \in \stackrel{\circ}{D}$ the preceding arguments apply equally well to $V_{\tau}^{(2)}$, and we may conclude that $T_{\imath \tau}^{(1)}$ is an analytic family of compact operators in $\stackrel{\circ}{D}$. However, since

$$
\begin{aligned}
{\left[V_{\mu+i \sigma}^{(2)} f\right](\xi) } & =(2 \pi)^{-n} \int_{\mathbb{R}^{n}} \frac{\left(1-\chi\left(\eta_{v}\right)\right) q_{0}(\xi-\eta, \eta-\sigma v+i \mu v) f(\eta)}{|\eta-\sigma v|^{2}-k^{2}-\mu^{2}+2 i \mu\left(\eta_{v}-\sigma\right)} d \eta \\
& =(2 \pi)^{-n} \cdot \int_{\mathbb{R}^{n}} \frac{\left(1-\chi\left(\eta_{v}+\sigma\right)\right) q_{0}(\xi-\eta-\sigma v, \eta+i \mu \nu) f(\eta+\sigma v)}{|\eta|^{2}-k^{2}-\mu^{2}+2 i \mu \eta_{v}} d \eta
\end{aligned}
$$

we need to show that $V_{\mu+i \sigma}^{(2)}$ extends continuously to $\mu=0$ from $\mu>0$. Since $\eta_{v}$ does not vanish on the support of $\left(1-\chi\left(\eta_{v}+\sigma\right)\right)$ for $|\sigma|<\varepsilon_{1} / 2$, we can again deform the integration in $r$ into $\operatorname{Im} r>0$ for $\eta_{v}>0$ and into $\operatorname{Im} r<0$ for $\eta_{v}<0$, using the piecewise linear contour $\Gamma^{\prime}$ connecting 0 to $\varepsilon / 2+i \varepsilon / 2$ sgn $\eta_{v}$ to $3 k / 2+i \varepsilon / 2$ sgn $\eta_{v}$ to $3 k / 2$ to $\infty$. Then for $r \in \Gamma^{\prime}$ and $0 \leqq \mu \leqq \varepsilon_{1 / 2}$,

$$
\begin{aligned}
\left|\eta \cdot \eta-k^{2}-\mu^{2}+2 i \mu \eta_{v}\right|^{-1} & =\left|r^{2}+\eta_{v}^{2}-k^{2}-\mu^{2}+2 i \mu \eta_{v}\right|^{-1} \\
& \leqq C_{k, \varepsilon / 2}\left(|r|^{2}+\left|\eta_{v}-\left(\operatorname{sgn} \eta_{v}\right) k\right|\right)^{-1}
\end{aligned}
$$

because $r=\left(1+i \operatorname{sgn} \eta_{v}\right) t$ on the first segment of $\Gamma^{\prime}$ and $r^{2}=2 i\left(\operatorname{sgn} \eta_{v}\right) t^{2}$. Since $\left(|r|^{2}+\left|\eta_{v}-\left(\operatorname{sgn} \eta_{v}\right) k\right|\right)^{-1}$ is locally integrable with respect to $|r|^{n-2} d|r| d \eta_{v}$, we may argue as follows. Removing small disks about $\left(r, \eta_{v}\right)=(0, \pm k)$ in the integral defining $V_{\mu+i \sigma}^{(2)} f$, we get an operator to which our previous arguments apply. Since this operator differs in norm from $V_{\mu+l \sigma}^{(2)}$ by an amount which goes to zero with the radius of disks, uniformly for $0 \leqq \mu \leqq \varepsilon_{1} / 2$, we conclude that $V_{\mu+i \sigma}^{(2)}$ extends continuously to a compact operator on $\mu=0$. 
In Sect. 3 we will show that $I+T_{i \tau}^{(1)}$ is invertible on $H_{0, n+1}$ for $\tau \gg 0$. This implies immediately that it is invertible on $A_{n+1, \delta / 3}$, since the null space of $I+T_{i \tau}^{(1)}$ on $A_{n+1, \delta / 3}$ is a subspace of its nullspace on $H_{0, n+1}$. Therefore, by Proposition 1 the set $Z$ where $I+T_{i \tau}^{(1)}$ is not invertible is discrete in $\stackrel{\circ}{D}$ and closed of measure zero in $D \cap\{\operatorname{Re} \tau=0\}$. In particular, there is an open interval $I=\left(\sigma_{1}, \sigma_{2}\right) \subset\left(-\varepsilon_{1} / 2, \varepsilon_{1} / 2\right)$ such that $I+T_{\imath \tau}^{(1)}$ is invertible for $\tau=-i \sigma, \sigma \in I$. Hence

$$
h_{v}(\xi, \zeta, k, i \tau)=\left[\left(I+T_{i \tau}^{(1)}\right)^{-1} q_{0}(\cdot-\zeta, \zeta+i \tau v)\right](\xi)
$$

exists for $\tau \in D \backslash Z$ and is analytic in $(\xi, \zeta, \tau)$ on $S_{\delta / 2} \times S_{\delta / 2} \times \stackrel{\circ}{D} \backslash Z$.

Our goal is to recover $h_{v}(\xi, \zeta, k, i \tau)$ from the scattering data. To make the connection with scattering data we will need to use $\tau=-i \sigma$ and identify $h_{v}$ with a translate of $h_{v, \sigma}$. Since denominator $(\eta+i \tau v) \cdot(\eta+i \tau v)-k^{2}$ with $\tau=\mu-i \sigma$ goes to $\eta \cdot \eta+2 \sigma \eta_{v}+\sigma^{2}-k^{2}$ as $\mu \downarrow 0$, we can remove the contour deformation in the definition of $V_{\tau}^{(1)} f$. However, since the integration in $r$ is deformed into the upper half-plane when $\eta_{v}>0$ and the lower half-plane when $\eta_{v}<0$, we have

$$
\left[T_{\sigma}^{(1)} f\right](\xi)=(2 \pi)^{-n} \int_{\mathbb{R}^{n}} \frac{q_{0}(\xi-\eta, \eta+\sigma v) f(\eta)}{\eta \cdot \eta+2 \sigma \eta_{\nu}+\sigma^{2}-k^{2}+i 0 \eta_{v}} d \eta
$$

and for $\sigma \in I, h_{v}(\xi, \zeta, k, \sigma)$ is the unique solution in $A_{n+1, \delta / 3}$ to

$$
f(\xi, \zeta)+(2 \pi)^{-n} \int_{\mathbb{R}^{n}} \frac{q_{0}(\xi-\eta, \eta+\sigma v) f(\eta, \zeta)}{\eta \cdot \eta+2 \sigma \eta_{v}+\sigma^{2}-k^{2}+i 0 \eta_{v}} d \eta=-q_{0}(\xi-\zeta, \zeta+\sigma v) .
$$

Since the changes of variables $\eta \rightarrow \eta-\sigma v, \xi \rightarrow \xi-\sigma v$ and $\zeta \rightarrow \zeta-\sigma v$, transform Eq. (32) to (16), we conclude that $h_{v}(\xi-\sigma v, \zeta-\sigma v, k, \sigma)$ is the unique solution of (16) in $A_{n+1, \delta / 3}$ and hence for $\sigma \in I$,

$$
h_{v}(\xi-\sigma v, \zeta-\sigma v, k, \sigma)=h_{v, \sigma}(\zeta, \zeta, k) \text {. }
$$

Therefore, assuming the results of Sect. 3, we have proven the following theorem:

Theorem 2. The solution $h_{v}(\xi, \zeta, k, i \tau)$ of (27) exists for $\tau \in D \backslash Z$ and is analytic in $(\xi, \zeta, \tau)$ on $S_{\delta / 3} \times S_{\delta / 3} \times(\stackrel{\circ}{D} \backslash Z)$. The limiting values of $h_{v}(\xi, \zeta, k, i \tau)$ when $\tau \rightarrow-i \sigma$ satisfy (33), where $h_{v, \sigma}(\xi, \zeta, k)$ is the solution of (16).

Since the unique solvability of (16) in $A_{n+1, \delta / 3}$ implies the unique solvability of (24) in $C\left(S^{n-1}\right)$, we know that (24) has a unique solution for $\sigma \in I$. Hence, knowing the scattering amplitude $h(\xi, \zeta, k)$ for $|\xi|^{2}=|\zeta|^{2}=k^{2}$, we can find $h_{v, \sigma}(\xi, \zeta, k)$ for $|\xi|^{2}=|\zeta|^{2}=k^{2}$ and $\sigma \in I$, which translates (by (33)) to knowing $h_{v}(\xi, \zeta, k, \sigma)$ for $|\xi+\sigma v|^{2}=|\zeta+\sigma v|^{2}=k^{2}$, for $\sigma \in I$. Since $h_{v}(\xi, \zeta, k, i \tau)$ is analytic for $(\xi, \zeta, \tau) \in$ $S_{\delta / 3} \times S_{\delta / 3} \times(\stackrel{\circ}{D} \backslash Z)$ with a continuous extension to $S_{\delta / 3} \times S_{\delta / 3} \times(-i I)$, we can determine it on the variety

$$
(\xi+i \tau v) \cdot(\xi+i \tau v)=(\zeta+i \tau v) \cdot(\zeta+i \tau v)=k^{2}
$$

for $(\xi, \zeta, \tau) \in S_{\delta / 3} \times S_{\delta / 3} \times(\stackrel{\circ}{D} \backslash Z)$ by analytic continuation. 
Fix $l \in R^{n}, \mu \in R^{n}, n \geqq 3$, such that

$$
l \cdot v=0, \quad \mu \cdot v=0, \quad l \cdot \mu=0, \quad \mu \cdot \mu=1,
$$

and put

$$
\begin{aligned}
& \xi(s)=\frac{1}{2} l+s \mu, \\
& \zeta(s)=\frac{-1}{2} l+s \mu, \\
& z(s)=i \tau(s)=i \sqrt{s^{2}+\frac{1}{4} l \cdot l-k^{2}},
\end{aligned}
$$

$s \geqq s_{0}, s_{0}$ large. We have that $h_{v}(\xi(s), \zeta(s), k, z(s))$ is analytic in $s$ for $s>s_{0}$ and

$$
(\xi(s)+i \tau(s) v) \cdot(\xi(s)+i \tau(s) v)=(\zeta(s)+i \tau(s) v) \cdot(\zeta(s)+i \tau(s) v)=k^{2} .
$$

Hence $h_{v}(\xi(s), \zeta(s), k, z(s))$ is known for $s>s_{0}$.

Remark 1. In the case $A(x)=0$ the operator $T_{i \tau}^{(1)}$ has a small norm in $H_{\zeta, n+1}$ (see Proposition 4) when $\tau>0$ is large. Substituting $\xi=\xi(s), \zeta=\zeta(s), z=z(s)=i \tau(s)$ in (27) and passing to the limit when $s \rightarrow+\infty$, we obtain that the integral in (27) tends to zero, and we can recover

$$
\hat{V}(l)=\lim _{s \rightarrow \infty} h_{v}(\xi(s), \zeta(s), k, z(s)) .
$$

Thus we obtain an alternate proof of R. Novikov's result [8].

\section{Solution of an Integral Equation}

In this section we set $z=i \tau$ and only consider $\tau$ real and positive.

In order to solve the integral equation (27) when $\tau$ is large and positive we will pass to an equivalent differential equation. Let

$$
v_{v}(x, \zeta, k, z)=(2 \pi)^{-n} \int_{\mathbb{R}^{n}} \frac{h_{v}(\eta, \xi, k, z) e^{i x \cdot \eta}}{(\eta+z v) \cdot(\eta+z v)-k^{2}} d \eta, \quad z=i \tau, \tau>0 .
$$

Then $v_{v}$, satisfies the differential equation

$$
\begin{gathered}
{\left[(-i \partial / \partial x+z v)^{2}-k^{2}+2 A(x) \cdot(-i \partial / \partial x+z v)+q(x)\right] v_{v}} \\
=-2(\zeta+z v) \cdot A(x) e^{i x \cdot \zeta}-q(x) e^{i x \cdot \zeta} .
\end{gathered}
$$

Our strategy will be to construct solutions of the equation

$$
\left[(-i \partial / \partial x+z v)^{2}-k^{2}+2 A(x) \cdot(-i \partial / \partial x+z v)+q(x)\right] v=f
$$

for all $f$ in the Banach space $H_{0, n+1}\left(R^{n}\right)$, where $H_{0, N}\left(R^{n}\right)$ is defined as the closure of $C_{0}^{\infty}\left(R^{n}\right)$ in the norm, $\|f\|_{0, N}=\sup _{\xi}(1+|\xi|)^{N}|\hat{f}(\xi)|$, i.e. $H_{0, N}$ is the Fourier transform of $H_{0, N}$. Then

$$
h(\xi)=\int_{\mathbb{R}^{n}}\left((-i \partial / \partial x+z v)^{2}-k^{2}\right) v(x) e^{-i x \cdot \xi} d x
$$


will be a solution of (27) with the inhomogeneous term replaced by $\hat{f}(\xi)$, i.e.

$$
h(\xi)+(2 \pi)^{-n} \int_{\mathbb{R}^{n}} \frac{q_{0}(\xi-\eta, \eta+z v) h(\eta)}{(\eta+z v) \cdot(\eta+z v)-k^{2}} d \eta=\hat{f}(\xi),
$$

and we will show that $h \in H_{0, n+1}$. Thus we can conclude that $I+T_{l \tau}^{(1)}$ (see (28)) maps $H_{0, n+1}$ onto $H_{0, n+1}$ for $\tau \gg 0$. Since $T_{i \tau}^{(1)}$ is also compact on $H_{0, n+1}$ for $\tau>0$, it follows that $I+T_{l \tau}^{(1)}$ is invertible on $H_{0, n+1}$ for $\tau \gg 0$, and (27) is uniquely solvable in $H_{0, n+1}$, when $\tau$ is sufficiently large positive.

We will look for a solution of $\left(37^{\prime}\right)$ in the form

$$
v(x, \zeta, z)=(2 \pi)^{-n} \int_{\mathbb{R}^{n}} \frac{c(x, \eta, z) \tilde{g}(\eta, \zeta, z) e^{i x \cdot \eta}}{(\eta+z v) \cdot(\eta+z v)-k^{2}} d \eta,
$$

where $z=i \tau, \tau>0$. Here $g(x, \zeta, z)$ is the new unknown and $\tilde{g}(\eta, \zeta, z)$ is its Fourier transform in the first variable. The factor $c(x, \eta, z)$ will be chosen so that the analogue of Eq. (27) for $\tilde{g}$ will not have the unbounded terms in $q_{0}(\xi-\eta, \eta+z v)$. For this reason we choose $c(x, \eta, z)$ as a solution of the transport equation

$$
-2 i \frac{\partial c}{\partial x} \cdot(\eta+z v)+2 A(x) \cdot(\eta+z v) \chi_{1}(\eta, z) c=0
$$

of the form $c=\exp \left(-i \chi_{1} \varphi\right)$. Thus $\varphi$ must satisfy

$$
(\eta+z v) \cdot \frac{\partial \varphi}{\partial x}=A(x) \cdot(\eta+z v)
$$

and we choose

$$
\varphi=(2 \pi)^{-n} \int_{\mathbb{R}^{n}} \frac{\hat{A}(\xi) \cdot(\eta+z v) e^{i x} \cdot \xi}{i \xi \cdot(\eta+z v)} d \xi .
$$

The function $\chi_{1}(\eta, z)$ is (40) is a cutoff to a neighborhood of $(\eta+z v) \cdot(\eta+$ $z v)=k^{2}$. The cancellation of unbounded terms is not needed outside this neighborhood, and it is convenient to have $c \equiv 1$ there. We choose $\chi(t) \in C_{0}^{\infty}(R)$ such that $\chi(t) \geqq 0, \chi(t)=1$ on $|t|<\varepsilon / 2$ and $\chi(t)=0$ on $|t|>\varepsilon$, and define

$$
\chi_{1}(\eta, z)=\chi\left(\frac{\left|(\eta+z v) \cdot(\eta+z v)-k^{2}\right|}{|\eta|^{2}+\tau^{2}+k^{2}}\right) .
$$

Since, setting $\eta_{v}=\eta \cdot v$,

$$
\left|(\eta+z v) \cdot(\eta+z v)-k^{2}\right|=\left(\left(|\eta|^{2}-\tau^{2}-k^{2}\right)^{2}+4 \tau^{2} \eta_{v}^{2}\right)^{1 / 2},
$$

it follows that on the support of $\chi_{1}$

$$
\varepsilon\left(|\eta|^{2}+\tau^{2}+k^{2}\right) \geqq\left.|| \eta\right|^{2}-\left(\tau^{2}+k^{2}\right) \mid,
$$

and hence

$$
\left(\frac{1-\varepsilon}{1+\varepsilon}\right)|\eta|^{2}<\tau^{2}+k^{2}<\left(\frac{1+\varepsilon}{1-\varepsilon}\right)|\eta|^{2} .
$$


Setting $\eta^{\prime}=\eta-(\eta \cdot v) v,(42)$ also implies that on the support of $\chi_{1}$,

$$
2 \varepsilon\left(\left|\eta^{\prime}\right|^{2}+\eta_{v}^{2}+\tau^{2}+k^{2}\right) \geqq\left.|| \eta^{\prime}\right|^{2}+\eta_{v}^{2}-\tau^{2}-k^{2}|+2 \tau| \eta_{v} \mid
$$

and hence, using (43),

$$
\begin{aligned}
(1+2 \varepsilon)\left|\eta^{\prime}\right|^{2} & \geqq(1-2 \varepsilon)\left(\tau^{2}+k^{2}\right)-(1+2 \varepsilon) \eta_{v}^{2}+2 \tau\left|\eta_{v}\right| \\
& \geqq(1-2 \varepsilon)\left(\tau^{2}+k^{2}\right)+\left(2\left(\frac{1-\varepsilon}{1+\varepsilon}\right)^{1 / 2} \frac{\tau}{\left(\tau^{2}+k^{2}\right)^{1 / 2}}-(1+2 \varepsilon)\right) \eta_{v}^{2} .
\end{aligned}
$$

Thus, choosing $\varepsilon$ sufficiently small and $\tau_{0}$ sufficiently large, we have for $\tau \geqq \tau_{0}$,

$$
\left(\tau^{2}+k^{2}\right)+\eta_{v}^{2} \leqq C_{\varepsilon}\left|\eta^{\prime}\right|^{2}
$$

on support $\chi_{1}$.

We will need some detailed estimates on $\varphi$. The behavior of $\varphi$ in the $x$-variables is strongly dependent on $\eta$. We introduce $\mu=\eta^{\prime} /\left|\eta^{\prime}\right|$, and use the orthogonal expansion $x=x_{1} v+x_{2} \mu+x_{\perp}$, where $x_{\perp}$ is the projection of $x$ on the orthogonal complement of span $\{v, \eta\}$.

Proposition 2. Assume that $B(x)$ is a vector-valued function satisfying (3) and define

$$
\psi(x, \eta+z v)=(2 \pi)^{-n} \int_{\mathbb{R}^{n}} \frac{\hat{B}(\xi) \cdot(\eta+z v)}{\xi \cdot(\eta+z v)} e^{i x} \cdot \xi \xi .
$$

Then for $(\eta, z) \in \operatorname{supp} \chi_{1}, \tau \geqq \tau_{0}$ and $|\alpha|+|\beta| \leqq P$ in $\left(3^{\prime}\right)$ one has

$$
\left|\frac{\partial^{|\alpha|+|\beta|} \psi}{\partial x^{\alpha} \partial \eta^{\beta}}\right| \leqq C_{\alpha \beta} \tau^{-|\beta|} e^{-\frac{\partial}{2}\left|x_{\perp}\right|}
$$

Proof. By contour integration one computes

$$
(2 \pi)^{-2} \int_{\mathbb{R}^{2}} \frac{e^{l\left(x_{1} \xi_{1}+x_{2} \xi_{2}\right)}}{\xi \cdot(\eta+z v)} d \xi_{1} d \xi_{2}=\frac{1}{2 \pi} \frac{1}{\left|\eta^{\prime}\right| x_{1}-\left(\eta_{v}+z\right) x_{2}} .
$$

Thus

$$
\psi(x, \eta+z v)=\frac{1}{2 \pi} \int_{\mathbb{R}^{2}} \frac{B\left(x-y_{1} v-y_{2} \mu\right) \cdot(\eta+z v)}{\left|\eta^{\prime}\right| y_{1}-\left(\eta_{v}+z\right) y_{2}} d y,
$$

and, using $\left(3^{\prime}\right)$, for $|\alpha| \leqq P$,

$$
\left|\frac{\partial^{|\alpha|} \psi}{\partial x^{\alpha}}(x, \eta+z v)\right| \leqq \int_{\mathbb{R}^{2}} \frac{C e^{-\delta\left|\left(x_{1}-y_{1}\right) v+\left(x_{2}-y_{2}\right) \mu+x_{\perp}\right|}|\eta+z v|}{\| \eta^{\prime}\left|y_{1}-\left(\eta_{v}+z\right) y_{2}\right|} d y .
$$

Since (43) and (44) imply that

$$
\begin{aligned}
\| \eta^{\prime}\left|y_{1}-\left(\eta_{v}+z\right) y_{2}\right| & =\left(\left(\left|\eta^{\prime}\right| y_{1}-\eta_{v} y_{2}\right)^{2}+\tau^{2} y_{2}^{2}\right)^{1 / 2} \\
& \geqq C \tau\left(y_{1}^{2}+y_{2}^{2}\right)^{1 / 2}=C \tau|y|,
\end{aligned}
$$


it follows from (43) and (47) that

$$
\left|\frac{\partial^{|\alpha|} \psi}{\partial x^{\alpha}}(x, \eta+z v)\right| \leqq C_{\alpha} e^{-\frac{\partial}{2}\left|x_{\perp}\right|}
$$

for $|\alpha| \leqq P$, where $C_{\alpha}$ is independent of $\eta$ and $z$.

To estimate $\eta$ derivatives of $\psi$ we first observe that (48) implies

$$
\left|\frac{\partial}{\partial \eta_{j}}\left(\frac{1}{\left|\eta^{\prime}\right| y_{1}-\left(\eta_{v}+z\right) y_{2}}\right)\right|=\left|\frac{\frac{\partial\left|\eta^{\prime}\right|}{\partial \eta_{1}} y_{1}-\frac{\partial \eta_{v}}{\partial \eta_{j}} y_{2}}{\left(\left|\eta^{\prime}\right| y_{1}-\left(\eta_{v}+z\right) y_{2}\right)^{2}}\right| \leqq \frac{C}{\tau^{2}|y|} .
$$

Thus, differentiating (46),

$$
\begin{aligned}
\left|\frac{\partial \psi}{\partial \eta_{j}}\right| & \leqq \frac{C}{\tau} \int_{\mathbb{R}^{2}} \frac{\left|B\left(x-y_{1} v-y_{2} \mu\right)\right| d y}{|y|}+\frac{C}{\tau} \int_{\mathbb{R}^{2}}\left|\frac{\partial B}{\partial x}\left(x-y_{1} v-y_{2} \mu\right)\right| d y \\
& \leqq \frac{C}{\tau} e^{-\frac{\delta}{2}\left|x_{\perp}\right|} .
\end{aligned}
$$

Repeating the same argument and noting that $\partial^{|\gamma|} / \partial \eta^{\gamma}\left(\left|\eta^{\prime}\right| y_{1}-\left(\eta_{v}+z\right) y_{2}\right)^{-1}$ is homogeneous of degree -1 in $y$ for any $\gamma$, one concludes

$$
\left|\frac{\partial^{|\alpha|+|\beta|} \psi}{\partial x^{\alpha} \partial \eta^{\beta}}\right| \leqq \frac{C_{\alpha \beta}}{\tau^{|\beta|}} e^{-\frac{\delta}{2}\left|x_{\perp}\right|}
$$

for $|\alpha|+|\beta| \leqq P$ and $\tau \geqq \tau_{0}$ on the support of $\chi_{1}$.

To study $\varphi$ in (41) we will use Proposition 2. We introduce

$$
w=x_{1}-\left(\eta_{v}+z\right)\left|\eta^{\prime}\right|^{-1} x_{2} \quad \text { and } \quad w^{\prime}=y_{1}-\left(\eta_{v}+z\right)\left|\eta^{\prime}\right|^{-1} y_{2}
$$

and observe that

$$
\frac{1}{w-w^{\prime}}=\frac{1}{w\left(1-\frac{w^{\prime}}{w}\right)}=\sum_{k=0}^{N} \frac{\left(w^{\prime}\right)^{k}}{w^{k+1}}+\frac{\left(w^{\prime}\right)^{N+1}}{w^{N+1}\left(w-w^{\prime}\right)} .
$$

Then we can write (46) with $B$ replaced by $A / i$ in the form

$$
\begin{aligned}
\varphi(x, \eta+z v) & =\frac{1}{2 \pi i} \int_{\mathbb{R}^{2}} \frac{A\left(y_{1} v+y_{2} \mu+x_{\perp}\right) \cdot(\eta+z v)}{\left|\eta^{\prime}\right|\left(x_{1}-y_{1}\right)-\left(\eta_{v}+z\right)\left(x_{2}-y_{2}\right)} d y \\
& =\frac{1}{2 \pi\left|\eta^{\prime}\right| i_{\mathbb{R}^{2}}} \frac{A\left(y_{1} v+y_{2} \mu+x_{\perp}\right) \cdot(\eta+z v)}{w-w^{\prime}} d y .
\end{aligned}
$$

Using (50) to expand (51), the remainder term in (50) contributes a term to $\varphi$ of the form

$$
\frac{1}{2 \pi i} \frac{1}{w^{N+1}} \int_{\mathbb{R}^{2}} \frac{B_{N}\left(x-y_{1} v-y_{2} \mu, \eta, z\right) \cdot(\eta+z v)}{\left|\eta^{\prime}\right| y_{1}-\left(\eta_{v}+z\right) y_{2}} d y,
$$

where $B_{N}(x, \eta, z)=\left(x_{1}-\left(\eta_{v}+z\right)\left|\eta^{\prime}\right|^{-1} x_{2}\right)^{N+1} A(x)$ satisfies (3) uniformly in $(\eta, z)$ on the support of $\chi_{1}$ for $\tau \geqq \tau_{0}$. The other terms in (50) contribute terms to $\varphi$ of the form

$$
\frac{1}{2 \pi i} \frac{1}{w^{k+1}} \int_{\mathbb{R}^{2}}\left|\eta^{\prime}\right|^{-1} A\left(x^{\perp}+y_{1} v+y_{2} \mu\right) \cdot(\eta+z v)\left(w^{\prime}\right)^{k} d y .
$$


Thus we see that for any $N \geqq 0$, when $(\eta, z)$ is in the support of $\chi_{1}$ and $\tau \geqq \tau_{0}$,

$$
\varphi=\sum_{k=1}^{N-1} w^{-k} b_{k}\left(x_{\perp}, \eta, z\right)+w^{-N} b_{N},
$$

where $\psi=b_{N}$ satisfies (45) and $b_{k}\left(x_{\perp}, \eta, z\right)$ is exponentially decreasing in $x_{\perp}$ together with its derivatives up to order $P$ uniformly in $(\eta, z)$.

Substituting (39) into $\left(37^{\prime}\right)$ and using (40), we obtain

$$
C(x, D, z) g+T_{1} g+T_{2} g+T_{3} g=f,
$$

where

$$
\begin{aligned}
& {\left[T_{1} g\right](x)=(2 \pi)^{-n} \int_{\mathbb{R}^{n}} \frac{\left(-2 i A \cdot \frac{\partial c}{\partial x}+q c\right) \hat{g}(\eta) e^{l x} \cdot \eta}{(\eta+z v) \cdot(\eta+z v)-k^{2}} d \eta,} \\
& {\left[T_{2} g\right](x)=(2 \pi)^{-n} \int_{\mathbb{R}^{n}} \frac{(-\Delta c) \hat{g}(\eta) e^{l x} \cdot \eta d \eta}{(\eta+z v) \cdot(\eta+z v)-k^{2}},} \\
& {\left[T_{3} g\right](x)=(2 \pi)^{-n} \int_{\mathbb{R}^{n}} \frac{2\left(1-\chi_{1}\right) A \cdot(\eta+z v) c \hat{g}(\eta) e^{i x} \cdot \eta}{(\eta+z v) \cdot(\eta+z v)-k^{2}} d \eta,}
\end{aligned}
$$

and $C(x, D, z)$ is a pseudo-differential operator with symbol $c(x, \eta, z)$.

In Sects. 4 and 5 we will need uniform estimates on the norms of the operators $e^{-i x \cdot \zeta} T_{j} e^{l x \cdot \zeta}, j=1,2,3$, and $e^{-i x \cdot \zeta} C e^{i x \cdot \zeta}$. Since multiplication by $e^{l x \cdot \zeta}$ is not bounded on $H_{0, N}$ (for $N>0$ ) and $\zeta \rightarrow \infty$, these estimates do not follow from estimates on the norms of the $T_{j}, j=1,2,3$ and $C$ on $H_{0, N}$. To prove what we will use later efficiently we are going to equip $H_{0, N}$ with a family of norms, \|\|$_{\zeta, N}$ so that estimates in these norms uniform in $\zeta$ will imply the needed estimates for Sects. 4 and 5. We will refer to $H_{0, N}$ with the norm \|\|$_{\zeta, N}$ as " $H_{\zeta, N}$."

Proposition 3. Let $H_{\zeta, N}\left(R^{n}\right)$ be the closure of $C_{0}^{\infty}\left(R^{n}\right)$ in the norm $\|f\|_{\zeta, N}=$ $\sup _{\mathbb{R}^{n}}(1+|\xi-\zeta|)^{N}|\hat{f}(\xi)|$. Then $C(x, D, z)$ is invertible as an operator on $H_{\zeta, n+1}$ $\left(R^{n}\right)$ for $\tau$ sufficiently large.

Proof. Our approach here will be to show that $C(x, D)$ and the operator $C^{(-1)}(x, D)$ with the reciprocal symbol $e^{l \chi_{1} \varphi}$ are bounded on $H_{\zeta, n+1}$. Then the composition formula for pseudo-differential operators and Proposition 2 will be used to show

$$
C^{(-1)} C=I+T
$$

where the norm of $T$ on $H_{\zeta, n+1}$ goes to zero as $\tau \rightarrow \infty$ uniformly in $\zeta$.

The proof that $C$ and $C^{(-1)}$ are uniformly bounded on $H_{\zeta, n+1}$ uses only (52). Expanding $c(x, \eta, z)=\exp \left(-i \varphi \chi_{1}\right)$ in a Taylor series in $\varphi \chi_{1}$, it is clear that $c-1$ also has an expansion of the form (52) for $\tau \geqq \tau_{0}$. A linear transformation of $R^{n}$ takes $w$ in (52) to the standard complex variable $z=s+i$. Hence analytic functions of $w$ are annihilated by the pull-back of $\partial / \partial \bar{z}$ under this transformation which is 
$\frac{\partial}{\partial \bar{w}}=\frac{1}{2}\left(\frac{\partial}{\partial x_{2}}+\left(\eta_{v}+z\right)\left|\eta^{\prime}\right|^{-1} \frac{\partial}{\partial x_{1}}\right)$. From (52) we have $\left\|\left(\partial^{|\alpha|} / \partial x^{\alpha}\right) \partial c / \partial \bar{w}\right\|_{L^{1}\left(\mathbb{R}^{n}\right)} \leqq C$ for $|\alpha|<P$ uniformly on support $\chi_{1}$ for $\tau>\tau_{0}$. Thus setting $v_{0}=\partial c / \partial \bar{w}$,

$$
\left|\hat{v}_{0}(\xi, \eta, z)\right| \leqq C(1+|\xi|)^{-P+1}
$$

Thus, since $P \geqq n+2$, the inverse Fourier transform of $v_{0}(\xi)\left(\xi_{2}+\left(\eta_{v}+z\right)\right.$ $\left.\left|\eta^{\prime}\right|^{-1} \xi_{1}\right)^{-1}$ is continuous, tending to zero as $|x| \rightarrow 0$. Since $c$ is bounded, we conclude (by Liouville's theorem)

$$
\begin{aligned}
c(x, \eta, z) & =1+(2 \pi)^{-n} \int_{\mathbb{R}^{n}} \frac{2 \hat{v}_{0}(\xi) e^{i x \cdot \xi}}{i\left(\xi_{2}+\left(\eta_{1}+z\right)\left|\eta^{\prime}\right|^{-1} \xi_{1}\right)} d \xi \\
& =1+(2 \pi)^{-n} \int_{\mathbb{R}^{n}} \frac{2 \hat{v}_{0}(\xi)\left|\eta^{\prime}\right| e^{i x \cdot \xi}}{i \xi \cdot(\eta+z \nu)} d \xi .
\end{aligned}
$$

Using (55) and (56), given $C(x, D, z) g=h$, we have, setting $c_{1}=c-1$,

$$
\hat{h}(\xi)=\hat{g}(\xi)+\int_{\mathbb{R}^{n}} \tilde{c}_{1}(\xi-\eta, \eta, z) \hat{g}(\eta) d \eta,
$$

where $\tilde{c}_{1}(\xi, \eta, \zeta)$ has support in the support of $\chi_{1}$ and satisfies

$$
\left|\tilde{c}_{1}(\xi, \eta, z)\right| \leqq C\left|\eta^{\prime}\right|(1+|\xi|)^{-n-1}|\xi \cdot(\eta+z v)|^{-1} .
$$

Hence

$$
\begin{aligned}
\sup _{\xi}(1+|\xi-\zeta|)^{n+1}|\hat{h}(\xi)| \leqq & \left(1+\sup _{\xi, \zeta} \int_{\mathbb{R}^{n}}(1+|\xi-\zeta|)^{n+1}\left|\hat{c}_{1}(\xi-\eta, \eta, z)\right|\right. \\
& \left.(1+|\eta-\zeta|)^{-n-1} d \eta\right) \sup _{\xi}(1+|\xi-\zeta|)^{n+1}|\hat{g}(\xi)|,
\end{aligned}
$$

and the boundness of $C(x, D, z)$ on $H_{\zeta, n+1}\left(R^{n}\right)$ uniformly in $(\zeta, z)$ for $\tau \geqq \tau_{0}$ follows from (57) and the estimate

$$
\begin{aligned}
&(1+|\xi-\zeta|)^{n+1}(1+|\xi-\eta|)^{-n-1}(1+|\eta-\zeta|)^{-n-1} \\
& \leqq C\left((1+|\xi-\eta|)^{-n-1}+(1+|\eta-\zeta|)^{-n-1}\right) .
\end{aligned}
$$

To see that $C$ is invertible on $H_{\zeta, n+1}$ when $\tau$ is large, we recall that the integral remainder formula for Taylor series implies that the symbol of $C^{(-1)}(x, D, z) C(x, D, z)-I$ is given by

$$
r(x, \eta, z)=\sum_{|x|=1}(2 \pi)^{-n} \int_{\mathbb{R}^{n}}\left(\int_{0}^{1} e^{l x} \cdot \zeta \frac{\partial c^{-1}}{\partial \eta^{\alpha}}(x, \eta+t \zeta) \zeta^{\alpha} d t\right) \tilde{c}_{1}(\zeta, \eta) d \zeta .
$$

The analogue of (57) for $\partial c^{-1} / \partial \eta^{\alpha},|\alpha|=1$, is

$$
\left|\frac{\partial \tilde{c}^{-1}}{\partial \eta^{\alpha}}(\xi, \eta, z)\right| \leqq C \frac{\left|\eta^{\prime}\right|}{\tau}(1+|\xi|)^{-n-1}|\xi \cdot(\eta+z v)|^{-1} .
$$

We can now apply the argument, used above to show that $C(x, D, z)$ is bounded on $H_{\zeta, n+1}$, to $R(x, D, z)$. The superpositions in $\zeta$ and $\tau$ produce no new difficulties and 
the factor of $1 / \tau$ in the estimate for $\partial c^{-1} / \partial \eta^{\alpha}$ above makes $\|R(x, D)\|$ go to zero as $\tau \rightarrow \infty$. Thus $C$ is invertible for $\tau$ sufficiently large.

Proposition 4. The norms of the operators $T_{1}(\tau), T_{2}(\tau)$ and $T_{3}(\tau)$ on $H_{\zeta, n+1}\left(R^{n}\right)$ tend to zero as $\tau \rightarrow \infty$ uniformly in $\zeta$.

Proof. Let $\tilde{T}_{k}(\xi-\eta, \eta, z)$ be the kernel of the Fourier transform of $T_{k}, k=1,2,3$, i.e.

$$
\widehat{T_{k} g}(\xi)=\int_{\mathbb{R}^{n}} \tilde{T}_{k}(\xi-\eta, \eta, z) \hat{g}(\eta) d \eta
$$

In order to show that the norm of $T_{k}$ on $H_{\zeta, n+1}\left(R^{n}\right)$, is arbitrarily small for $\tau$ large uniformly in $\zeta$, it suffices to prove that

$$
\sup _{\zeta, \zeta, \mathbb{R}^{n}}(1+|\xi-\zeta|)^{n+1}\left|\tilde{T}_{k}(\xi-\eta, \eta, z)\right|(1+|\eta-\zeta|)^{-n-1} d \eta \leqq \frac{C}{\tau} \log \tau
$$
Hence

On the support of $1-\chi_{1}$ we have $\left|(\eta+z v) \cdot(\eta+z v)-k^{2}\right| \geqq \frac{\varepsilon}{2}\left(|\eta|^{2}+\tau^{2}+k^{2}\right)$.

$$
\left|\tilde{T}_{3}(\xi-\eta, \eta, z)\right| \leqq C(1+|\xi-\eta|)^{-n-1} \frac{|\eta+z v|}{|\eta|^{2}+\tau^{2}+k^{2}} \leqq \frac{C}{\tau}(1+|\xi-\eta|)^{-n-1}
$$

and (59) for $k=3$ follows from (58).

To estimate $\tilde{T}_{1}$ we note that (42) implies that for all $(\eta, z)$,

$$
\begin{aligned}
& \mid \eta+z v) \cdot(\eta+z v)-k^{2} \mid \geqq \frac{1}{2}\left(\left.|| \eta\right|^{2}-\left(\tau^{2}+k^{2}\right)|+2 \tau| \eta_{v} \mid\right) \\
& \quad=\frac{1}{2}\left(|| \eta\left|-\left(\tau^{2}+k^{2}\right)^{1 / 2}\right||| \eta\left|+\left(\tau^{2}+k^{2}\right)^{1 / 2}\right|+2 \tau\left|\eta_{v}\right|\right) \\
& \quad \geqq \frac{\tau}{2}\left(|| \eta\left|-\left(\tau^{2}+k^{2}\right)^{1 / 2}\right|+\left|\eta_{v}\right|\right) .
\end{aligned}
$$

Since $c-1$ has an expansion of the form (52), qc and $A \cdot \frac{\partial c}{\partial x}$ satisfy (3) with constants uniform in $(\eta, z)$ for $\tau>\tau_{0}$. Thus, from (58) and (60),

$$
\begin{aligned}
& \sup _{\xi, \zeta \mathbb{R}^{n}}(1+|\xi-\zeta|)^{n+1}\left|\tilde{T}_{1}(\xi-\eta, \eta, z)\right|(1+|\eta-\zeta|)^{-n-1} d \eta \\
& \leqq \frac{C}{\tau} \sup _{\xi, \zeta \mathbb{R}^{n}} \frac{(1+|\xi-\eta|)^{-n-1}+(1+|\eta-\zeta|)^{-n-1}}{|| \eta\left|-\left(\tau^{2}+k^{2}\right)^{1 / 2}\right|+\left|\eta_{v}\right|} d \eta \\
& \quad \leqq \frac{2 C}{\tau} \sup _{\xi} \int_{\mathbb{R}^{n}} \frac{(1+|\xi-\eta|)^{-n-1}}{|| \eta\left|-\left(\tau^{2}+k^{2}\right)^{1 / 2}\right|+\left|\eta_{v}\right|} d \eta .
\end{aligned}
$$


Setting $R=\left(\tau^{2}+k^{2}\right)^{1 / 2}, \eta=R \zeta$ and $l(\zeta)=\left((|\zeta|-1)^{2}+\zeta_{v}^{2}\right)^{1 / 2}$ in the last line of (61), this gives

$$
\begin{aligned}
& \sup _{\zeta, \zeta} \int_{\mathbb{R}^{n}}(1+|\xi-\zeta|)^{n+1}\left|\tilde{T}_{1}(\xi-\eta, \eta, z)\right|(1+|\eta-\zeta|)^{-n-1} d \eta \\
& \leqq \frac{C}{\tau} \sup _{\zeta} \int_{\mathbb{R}^{n}}(1+|\xi-R \zeta|)^{-n-1}(l(\zeta))^{-1} R^{n-1} d \zeta \\
& \leqq \frac{C}{\tau}\left[\sup _{\xi} \int_{\mathbb{R}^{n}}(1+|\xi-R \zeta|)^{-n-1} R^{n-1} d \zeta\right. \\
& \left.+\sup _{\zeta} \int_{l(\zeta)<\varepsilon_{0}}(1+|\xi-R \zeta|)^{-n-1}(l(\zeta))^{-1} R^{n-1} d \zeta\right] \text {. } \\
& \leqq \frac{C}{\tau}\left[\frac{1}{R}+R^{n-1} \sup _{\xi} \int_{l(\zeta)<\varepsilon_{0}}\left(1+|\xi-R \zeta|^{-n-1}(l(\zeta))^{-1} d \zeta\right] .\right.
\end{aligned}
$$

Here $\varepsilon_{0}$ is any fixed constant, and we assume $\varepsilon_{0} \ll 1$. Since $\tau \sim R$ for $\tau>\tau_{0}$, it suffices to show

$$
\tau^{n-1} \sup _{\xi} \int_{l(\zeta)<\varepsilon_{0}}(1+\tau|\xi-\zeta|)^{-n-1}(l(\zeta))^{-1} d \zeta<C
$$

for $\tau>\tau_{0}$ to conclude that (59) holds for $k=1$.

To prove (62) we note first that when $\left|\xi^{\prime}\right|<\frac{1}{2}$,

$$
\int_{l(\zeta)<\varepsilon_{0}}(1+\tau|\xi-\zeta|)^{-n-1}(l(\zeta))^{-1} d \zeta \leqq \int_{l(\zeta)<\varepsilon_{0}}\left(1+c_{0} \tau\right)^{-n-1}(l(\zeta))^{-1} d \zeta,
$$

where $c_{0}=\min _{l(\zeta)<\varepsilon_{0}}|\xi-\zeta|>0$, and (62) holds.

To establish (62) for $\left|\xi^{\prime}\right|>\frac{1}{2}$ we will use spherical coordinates in the hyperplane $\zeta \cdot v=0$ with $r=\left|\zeta^{\prime}\right|$ and polar angle $\theta=\cos ^{-1}\left(\frac{\zeta^{\prime}}{\left|\zeta^{\prime}\right|} \cdot \frac{\zeta^{\prime}}{\left|\zeta^{\prime}\right|}\right)$. Then we have $d \zeta=r^{n-2} d r d \omega d \zeta_{v}$, where $d \omega$ is the volume form on $S^{n-2}$, and we also have

$$
\begin{aligned}
|\zeta-\xi| & =\left(r^{2}-2\left|\xi^{\prime}\right| r \cos \theta+\left|\xi^{\prime}\right|^{2}+\left(\zeta_{v}-\xi_{v}\right)^{2}\right)^{1 / 2} \\
& \geqq \frac{1}{2}\left(\left(\left(r-\left|\xi^{\prime}\right| \cos \theta\right)^{2}+\left(\zeta_{v}-\xi_{v}\right)^{2}\right)^{1 / 2}+\left|\xi^{\prime}\right||\sin \theta|\right) .
\end{aligned}
$$

Likewise, there is $c>0$ such that

$$
l(\zeta) \geqq c\left((r-1)^{2}+\zeta_{v}^{2}\right)^{1 / 2} .
$$

Now we consider $v=\left(r-1, \zeta_{v}\right)$ and $v_{0}=\left(\left|\xi^{\prime}\right| \cos \theta-1, \xi_{v}\right)$ as vectors in $R^{2}$ and use \|\| to denote the norm on $R^{2}$. From (63) and (64) we have

$$
\begin{aligned}
& \int_{l(\zeta)<\varepsilon_{0}}(1+\tau|\xi-\zeta|)^{-n-1}(l(\zeta))^{-1} d \zeta \\
& \quad \leqq C \int_{\mathbb{R}^{2} \times S^{n-2}} \frac{\left(1+\tau\left(\left\|v-v_{0}\right\|+|\sin \theta|\right)\right)^{-n-1}}{\|v\|} d r d \zeta_{1} d \omega .
\end{aligned}
$$


We split the integral over $R^{2} \times S^{n-2}$ into an integral over $\left\{\zeta:\|v\| \geqq\left\|v-v_{0}\right\|\right\}$ in which we replace $\|v\|$ by $\left\|v-v_{0}\right\|$ and an integral over $\left\{\zeta:\|v\|<\left\|v-v_{0}\right\|\right\}$ in which we replace $\left\|v-v_{0}\right\|$ by $\|v\|$. Since the two integrands that are produced this way differ only by a translation in the $\left(r, \zeta_{v}\right)$-plane, we have the estimate

$$
\begin{aligned}
& \int_{l(\zeta)<i_{0}}(1+\tau|\xi-\zeta|)^{-n-1}(l(\zeta))^{-1} d \zeta \\
& \leqq C \int_{\mathbb{R}^{2} \times S^{n-2}} \frac{\left(1+\tau\left(\left(s^{2}+t^{2}\right)^{1 / 2}+|\sin \theta|\right)\right)^{-n-1}}{\left(s^{2}+t^{2}\right)^{1 / 2}} d s d t d \omega \\
& \leqq C \int_{0}^{\infty} \int_{S^{n-2}}(1+\tau(u+|\sin \theta|))^{-n-1} d u d \omega \\
& \leqq C \int_{0}^{\infty \pi / 2} \int_{0}^{\infty}(1+\tau(u+\theta))^{-n-1} \theta^{n-3} d u d \theta
\end{aligned}
$$

and, setting $\tau u=r, \tau \theta=s$, we have

$$
\int_{l(\zeta)<\varepsilon_{0}}(1+\tau|\xi-\zeta|)^{-n-1}(l(\zeta))^{-1} d \zeta \leqq \tau^{-n+1} C \int_{0}^{\infty} \int_{0}^{\infty}(1+r+s)^{-n-1} s^{n-3} d r d s .
$$

Thus, since the integral is finite, we have (62), and (59) holds for $k=1$, in the stronger form

$$
\sup _{\xi, \zeta} \int_{\mathbb{R}^{n}}(1+|\xi-\zeta|)^{n+1}\left|\tilde{T}_{1}(\xi-\eta, \eta, z)\right|(1+|\eta-\zeta|)^{-n-1} d \eta \leqq \frac{C}{\tau} .
$$

From (56) one sees that

$$
|\widetilde{\Delta} c(\xi-\eta, \eta)| \leqq C(1+|\xi-\eta|)^{-P+3}\left|\eta^{\prime} \|(\xi-\eta) \cdot(\eta+z v)\right|^{-1}
$$

and hence

$$
\left|\tilde{T}_{2}(\xi-\eta, \eta, z)\right| \leqq \frac{C(1+|\xi-\eta|)^{-P+3}\left|\eta^{\prime}\right|}{|(\xi-\eta) \cdot(\eta+z v)|(\eta+z v) \cdot(\eta+z v)-k^{2} \mid},
$$

and by the reasoning that leads to (61), we have (note $P \geqq n+4$ is needed):

$$
\begin{aligned}
& \sup _{\xi, \zeta \mathbb{R}^{n}}(1+|\xi-\zeta|)^{n+1}\left|\tilde{T}_{2}(\xi-\eta, \eta, z)\right|(1+|\eta-\zeta|)^{-n-1} d \eta \\
& \quad \leqq \frac{C}{\tau} \sup _{\xi} \int_{\mathbb{R}^{n}} \frac{(1+|\xi-\eta|)^{-n-1}\left|\eta^{\prime}\right| d \eta}{|(\xi-\eta) \cdot(\eta+i \tau \nu)|\left(|| \eta\left|-\left(\tau^{2}+k^{2}\right)^{1 / 2}\right|+\left|\eta_{v}\right|\right)} .
\end{aligned}
$$

Setting $R=\left(\tau^{2}+k^{2}\right)^{1 / 2}, \beta=\tau\left(\tau^{2}+k^{2}\right)^{-1 / 2}, \eta=R \zeta$ and $l(\zeta)=\left((|\zeta|-1)^{2}+\zeta_{v}^{2}\right)^{1 / 2}$, (65) becomes

$$
\begin{aligned}
& \sup _{\tilde{\zeta}, \zeta} \int_{\mathbb{R}^{n}}(1+|\xi-\zeta|)^{n+1}\left|\tilde{T}_{2}(\xi-\eta, \eta, z)\right|(1+|\eta-\zeta|)^{-n-1} d \eta \\
& \quad \leqq \frac{C}{\tau} R^{n-1} \sup _{\zeta} \int_{\mathbb{R}^{n}} \frac{(1+|\xi-R \zeta|)^{-n-1}\left|\zeta^{\prime}\right| d \zeta}{|(\xi-R \zeta) \cdot(\zeta+i \beta v)| l(\zeta)} \\
& \quad=\frac{C}{\tau} R^{n-2} \sup _{\zeta} \int_{\mathbb{R}^{n}} \frac{(1+R|\xi-\zeta|)^{-n-1}\left|\zeta^{\prime}\right| d \zeta}{\left(((\xi-\zeta) \cdot \zeta)^{2}+\beta^{2}\left(\xi_{v}-\zeta_{v}\right)^{2}\right)^{1 / 2} l(\zeta)} .
\end{aligned}
$$


Since $\beta \rightarrow 1$ as $\tau \rightarrow \infty$ and $\beta R=\tau$, to show $\left\|T_{2}\right\| \rightarrow 0$ as $\tau \rightarrow \infty$, it suffices to show for $\tau>\tau_{0}$ that

$$
\tau^{n-2} \sup _{\zeta} \int_{\mathbb{R}^{n}} \frac{(1+\tau|\xi-\zeta|)^{-n-1}\left|\zeta^{\prime}\right| d \zeta}{\left(((\zeta-\zeta) \cdot \zeta)^{2}+\left(\xi_{v}-\zeta_{v}\right)^{2}\right)^{1 / 2} 1(\zeta)} \leqq C \log \tau
$$

When $1(\zeta)>\varepsilon_{0}$, the integrand in (66) is essentially the same as the one we considered for $T_{1}$ : note that $(\xi-\zeta) \cdot \zeta=|\zeta-\xi / 2|^{2}-|\xi / 2|^{2}$. Thus we again assume that $1(\zeta)<\varepsilon_{0} \ll 1$. We have

$$
\begin{aligned}
& \left(((\xi-\zeta) \cdot \zeta)^{2}+\left(\xi_{v}-\zeta_{v}\right)^{2}\right)^{1 / 2}>\frac{1}{2}\left(|(\xi-\zeta) \cdot \zeta|+\left|\xi_{v}-\zeta_{v}\right|\right) \\
& \quad=\frac{1}{2}\left(|| \zeta^{\prime}-\xi^{\prime} /\left.2\right|^{2}-\left|\xi^{\prime} / 2\right|^{2}+\zeta_{v}\left(\zeta_{v}-\xi_{v}\right)|+| \xi_{v}-\zeta_{v} \mid\right) \\
& \geqq \frac{1}{2}\left(|| \zeta^{\prime}-\xi^{\prime} /\left.2\right|^{2}-\left|\xi^{\prime} / 2\right|^{2}\left|+\left(1-\varepsilon_{0}\right)\right| \xi_{v}-\zeta_{v} \mid\right) .
\end{aligned}
$$

Again using the coordinates $r=\left|\zeta^{\prime}\right|, \theta=\cos ^{-1}\left(\zeta^{\prime} /\left|\zeta^{\prime}\right| \cdot \xi^{\prime} /\left|\xi^{\prime}\right|\right)$, we have

$$
\left|\zeta^{\prime}-\xi^{\prime} / 2\right|^{2}-\left|\xi^{\prime} / 2\right|^{2}=r^{2}-r\left|\xi^{\prime}\right| \cos \theta
$$

and

$$
\left(((\xi-\zeta) \cdot \zeta)^{2}+\left(\xi_{v}-\zeta_{v}\right)^{2}\right)^{1 / 2} \geqq c\left(\left(r-\left|\xi^{\prime}\right| \cos \theta\right)^{2}+\left(\xi_{v}-\zeta_{v}\right)^{2}\right)^{1 / 2}=c\left\|v-v_{0}\right\|,
$$

in the notation used earlier. Thus, using (64), for $\left|\xi^{\prime}\right| \leqq 1 / 2$,

$$
\int_{l(\zeta)<\varepsilon_{0}} \frac{(1+\tau|\xi-\zeta|)^{-n-1}\left|\zeta^{\prime}\right| d \zeta}{\left(((\xi-\zeta) \cdot \zeta)^{2}+\left(\xi_{v}-\zeta_{v}\right)^{2}\right)^{1 / 2} l(\zeta)} \leqq C \int_{l(\zeta)<\varepsilon_{0}} \frac{\left(1+c_{0} \tau\right)^{-n-1} d r d \zeta_{\nu} d \omega}{\left\|v-v_{0}\right\|\|v\|}
$$

and, since $\left|\xi^{\prime}\right| \leqq 1 / 2$ implies $\left\|v_{0}\right\| \geqq \frac{1}{2}$, this is bounded by $C \tau^{-n-1}$. Hence we may assume that $\left|\xi^{\prime}\right|>1 / 2$, and in this case (63) implies

$$
\begin{aligned}
& \int_{l(\zeta)<\varepsilon_{0}} \frac{(1+\tau|\xi-\zeta|)^{-n-1}\left|\zeta^{\prime}\right| d \zeta}{\left(((\xi-\zeta) \cdot \zeta)^{2}+\left(\xi_{v}-\zeta_{v}\right)^{2}\right)^{1 / 2} l(\zeta)} \\
& \quad \leqq C \int_{l(\zeta)<\varepsilon_{0}} \frac{\left(1+\tau\left(\left\|v-v_{0}\right\|+|\sin \theta|\right)\right)^{-n-1} d r d \zeta_{\nu} d \omega}{\left\|v-v_{0}\right\|\|v\|} \equiv I_{1} .
\end{aligned}
$$

Since (64) implies $\|v\|<\varepsilon_{0}$ when $l(\zeta)<\varepsilon_{0}$, we see that contribution to $I_{1}$ from integration over $\left\{\theta: \| v_{0}\left(\theta \| \geqq \frac{1}{2}\right\}\right.$ is bounded by $C \tau^{-n-1}$. Thus we may replace the domain of integration in $I_{1}$ by $\left\{l(\zeta)<\varepsilon_{0}\right\} \cap\left\{\left\|v_{0}\right\|<\frac{1}{2}\right\}$.

At this point the argument used for $T_{1}$ leads to divergent integrals, and we need to use the fact that the factors in the denominator only vanish simultaneously when 
$\left|\xi^{\prime}\right| \cos \theta=1$. To bound $I_{1}$, we set $z=\left(\left\|v_{0}(\theta)\right\|^{-1}\right) v$. Then

$$
\begin{aligned}
I_{1} & \leqq C \int_{\left.S^{n-2} \times\left\{\|z\| \leqq\left\|v_{0}\right\|^{-1}\right\}\right) \cap\left\{\left\|v_{0}\right\|<1 / 2\right\}} \frac{(1+\tau|\sin \theta|)^{-n-1}}{\left\|z-v_{0} /\right\| v_{0}\|\|\|z\|} d z d \omega \\
& \leqq C \int_{S^{n-2} \cap\left\{\left\|v_{0}\right\|<1 / 2\right\}}(1+\tau|\sin \theta|)^{-n-1} \log \left(\left\|v_{0}(\theta)\right\|^{-1}\right) d \omega \\
& \leqq C \int_{0}^{\pi / 2}(1+\tau \theta)^{-n-1} \max \left\{\log 2,-\log \left\|v_{0}(\theta)\right\|\right\} \theta^{n-3} d \theta \\
& \leqq C \tau^{2-n} \int_{0}^{\pi \tau / 2}(1+\beta)^{-n-1} \beta^{n-3} \max \left\{\log 2,-\log \left\|v_{0}\left(\frac{\beta}{\tau}\right)\right\|\right\} d \beta \\
I_{1} & \leqq c \tau^{2-n} \int_{0}^{\pi \tau / 2}(1+\beta)^{-n-1} \beta^{n-3} \max \left\{\log 2,-\log |1-| \xi^{\prime}\left|\cos \frac{\beta}{\tau}\right|\right\} d \beta .
\end{aligned}
$$

If $1 / 2 \leqq\left|\xi^{\prime}\right| \leqq 1$, then $|1-| \xi^{\prime}|\cos \beta / \tau| \geqq c_{0}^{2} \beta^{2} \tau^{-2}$ with $c_{0}$ independent of $\left|\xi^{\prime}\right|$. Hence, in this case $I_{1} \leqq c \tau^{2-n} \log \tau$ for $\tau$ large. If $\left|\xi^{\prime}\right|>1$, then $1-\left|\xi^{\prime}\right| \cos \theta=0$ has a unique solution $\theta_{0}$ in the interval $[0, \pi / 2]$ and we have

$$
|1-| \xi^{\prime}|\cos \theta| \geqq c_{0}^{2}\left(\theta-\theta_{0}\right)^{2}
$$

with $0<c_{0}<1$ and $c_{0}$ independent of $\left|\xi^{\prime}\right|$. Thus

$$
|1-| \xi^{\prime}|\cos \beta / \tau| \geqq \frac{c_{0}^{2}}{\tau^{2}}\left(\beta-\beta_{0}\right)^{2},
$$

where $\beta_{0}=\tau \theta_{0}$. Thus for $\tau>1$.

$$
\begin{aligned}
\max & \left\{\log 2,-\log |1-| \xi^{\prime}\left|\cos \frac{\beta}{\tau}\right|\right\} \\
& \leqq \log 2+2 \log \tau-2 \log c_{0}+2\left(-\log \left|\beta-\beta_{0}\right|\right)_{+}
\end{aligned}
$$

Combining (68) with (67) we see that $I_{1} \leqq C \tau^{2-n} \log \tau$ for $\tau$ large in this case also. Thus (66) holds and the proof of Proposition 4 is complete.

It follows from Propositions 3 and 4 that for $\tau \gg 0$ there exists a unique solution $g$ in $H_{0, n+1}$ of the integral equation (53), given by

$$
g=\left(I+(I+T)^{-1} C^{(-1)}\left(T_{1}+T_{2}+T_{3}\right)\right)^{-1}(I+T)^{-1} C^{(-1)} f,
$$

where $T$ is the operator in (54). Thus $v$, given by (39) with this choice of $g$, is a solution of $\left(37^{\prime}\right)$. Thus to complete the proof that (27) has a unique solution in $H_{0, n+1}\left(R^{n}\right)$ when $\tau \gg 0$, we need only show that $\check{h}$ given by

$$
\check{h}(x)=\left((-i \partial / \partial x+z v)^{2}-k^{2}\right) v
$$

is in $H_{0, n+1}$. From (39) we see that

$$
\check{h}=C g+T_{2} g+S g,
$$


where $T_{2}$ is the operator in (53) and

$$
\begin{aligned}
S g & =(2 \pi)^{-n} \int_{\mathbb{R}^{n}} \frac{-2 i \frac{\partial c}{\partial x} \cdot(\eta+z v) \hat{g}(\eta) e^{i x \cdot \eta}}{(\eta+z v) \cdot(\eta+z v)-k^{2}} d \eta \\
& =(2 \pi)^{-n} \int_{\mathbb{R}^{n}} \frac{-2 A(x) \cdot(\eta+z v) \chi_{1}(\eta, z) c \hat{g}(\eta) e^{i x \cdot \eta}}{(\eta+z v) \cdot(\eta+z v)-k^{2}} d \eta
\end{aligned}
$$

by (40). From (70) one sees that $S$ is an operator of the same type as $T_{1}$ in (53) with an additional factor of $\eta+z v$ in the numerator. However, since we showed that the norm of $T_{1}$ on $H_{\zeta, n+1}$ was $O\left(\tau^{-1}\right)$ uniformly in $\zeta$ for $\tau \rightarrow \infty$, and $|\eta+z v| \leqq c \tau$ on support $\chi_{1}$ (see (43)), it follows that $S$ is bounded on $H_{\zeta, n+1}$, uniformly in $(\zeta, \tau)$ for $\tau>\tau_{0}$. This completes the verification that $h_{v}(\xi, \zeta, k, i \tau) \in H_{\zeta, n+1}$.

\section{Recovering the Magnetic Field}

Proposition 5. Let $h_{v}(\xi, \zeta, k, z)$ be the unique solution of (27) in $H_{0, n+1}$ for $\tau \gg 0$, and let $g_{v}(x, \zeta, k, z)$ be the unique solution in $H_{0, n+1}$ of $(53)$ with $f=-(q(x)+$ $2(\zeta+z v)$. $A(x)) \exp (i x \cdot \zeta)$ for $\tau \gg 0$. Then

$$
h_{v}(\zeta, \zeta, k, z)=\tilde{g}_{v}(\xi, \zeta, k, z)
$$

when $(\xi+z v) \cdot(\xi+z v)-k^{2}=0$.

Proof. We have

$$
\begin{aligned}
v_{v}(x, \zeta, k, z) & =(2 \pi)^{-n} \int_{\mathbb{R}^{n}} \frac{h_{v}(\eta, \zeta, k, z) e^{i x \cdot \eta} d \eta}{(\eta+z v) \cdot(\eta+z v)-k^{2}} \\
& =(2 \pi)^{-n} \int_{\mathbb{R}^{n}} \frac{c(x, \eta, z) \tilde{g}_{v}(\eta, \zeta, k, z) e^{i x} \cdot \eta d \eta}{(\eta+z v) \cdot(\eta+z v)-k^{2}} .
\end{aligned}
$$

As we observed earlier $c_{1}=c(x, \eta, z)-1$ has an expansion of the form (52) for $\tau>\tau_{0}$. Thus, as in the proof of the bound on $T_{2}$ in Proposition 4 , we see that

$$
f(\xi, \zeta, k, z)=(2 \pi)^{-n} \int_{\mathbb{R}^{n}} \frac{\tilde{c}_{1}(\xi-\eta, \eta, z) \tilde{g}_{v}(\eta, \zeta, k, z) d \eta}{(\eta+z v) \cdot(\eta+z v)-k^{2}}
$$

belongs to $H_{0, n+1}$ as a function of $\xi$, and hence is continuous in $\xi$. Since the Fourier transform of (72) gives (a.e. in $\xi$ )

$$
\frac{h_{v}(\xi, \zeta, z)}{(\xi+z v) \cdot(\xi+z v)-k^{2}}=\frac{\tilde{g}_{v}(\xi, \zeta, k, z)}{(\xi+z v) \cdot(\xi+z v)-k^{2}}+f(\xi, \zeta, k, z),
$$

where $h_{v}$ and $\tilde{g}_{v}$ are also continuous in $\xi,(71)$ follows immediately. 
By Proposition 1 and the discussion following it we can recover $h_{v}(\xi(s), \zeta(s), k$, $z(s))$ from the scattering amplitude $h(k \Theta, k \omega, k)$. Recall (see (34), (35)) that given the orthogonal frame $\{v, \mu, l\}$ with $|\mu|=|v|=1$,

$$
\begin{aligned}
& \xi(s)=\frac{1}{2} l+s \mu, \\
& \zeta(s)=-\frac{1}{2} l+s \mu \\
& z(s)=i \tau(s)=i \sqrt{s^{2}+|l|^{2} / 4-k^{2}}
\end{aligned}
$$

for $s>s_{0}$. Since $(\xi(s)+z(s) v) \cdot(\xi(s)+z(s) v)-k^{2}=0$, it follows from Proposition 5 that $h(k \theta, k \omega, k)$ determines $\tilde{g}_{v}(\xi(s), \zeta(s), k, z(s))$ for $s>s_{0}$.

To recover the magnetic field we can begin with representation for $g_{v}$ given by (69) with $f=-(q(x)+2(\zeta+z v) \cdot A(x)) \exp (i x \cdot \zeta)$, take the Fourier transform in $x$, evaluate at $\xi=\xi(s), \zeta=\zeta(s), z=z(s)$ as in (73), divide by $z(s)$ and take the limit as $s \rightarrow \infty$. Since the norms of $T, T_{1}, T_{2}$ and $T_{3}$ on $H_{\zeta(s), n+1}$ go to zero and $\frac{1}{|z(s)|}\|f\|_{\zeta(s), n+1}$ is bounded as $s \rightarrow \infty$, it follows that $h(k \theta, k \omega, k)$ determines

$$
\begin{aligned}
\lim _{s \rightarrow \infty}(2 \pi)^{-n} \int_{\mathbb{R}^{n}} \int_{\mathbb{R}^{n}} & \frac{(-2)(\zeta(s)+z(s) v)}{z(s)} \cdot \hat{A}(\eta-\zeta(s)) \\
& \times e^{-i x \cdot(\xi(s)-\eta)+l \chi_{1}(\eta, z(s)) \varphi(x, \eta+z(s) v)} d \eta d x .
\end{aligned}
$$

Replacing $\eta-\zeta(s)$ by $\eta,(74)$ becomes

$$
\begin{aligned}
& \lim _{s \rightarrow \infty}(2 \pi)^{-n} \int_{\mathbb{R}^{n}} \int_{\mathbb{R}^{n}} \frac{(-2)(\zeta(s)+z(s) v)}{z(s)} \cdot \hat{A}(\eta) \\
& \times e^{i x} \cdot \eta-i x \cdot(\xi(s)-\zeta(s))+i \chi_{1}(\eta+\zeta(s), z(s)) \varphi(x, \eta+\zeta(s)+z(s) v) d \eta d x .
\end{aligned}
$$

By $(73) \xi(s)-\zeta(s)=l$ and $\lim _{s \rightarrow \infty}(\zeta(s)+z(s) v) / z(s)=v-i \mu$. Also (see definition of $\chi_{1}$ before (42))

$$
\lim _{s \rightarrow \infty} \chi_{1}(\eta+\zeta(s), z(s))=\chi(0)=1
$$

Finally

$$
\begin{aligned}
& \lim _{s \rightarrow \infty} \varphi(x, \eta+\zeta(s)+z(s) v)=\lim _{s \rightarrow \infty}(2 \pi)^{-n} \int_{\mathbb{R}^{n}} \frac{\hat{A}(\xi) \cdot(\eta+\zeta(s)+z(s) v) e^{i x} \cdot \xi}{i \xi \cdot(\eta+\zeta(s)+z(s) v)} d \xi \\
& \quad=(2 \pi)^{-n} \int_{\mathbb{R}^{n}} \frac{\hat{A}(\xi) \cdot(\mu+i v)}{i \xi \cdot(\mu+i v)} e^{i x} \cdot \xi \xi \equiv \varphi(x, \mu+i v) .
\end{aligned}
$$


Hence the limit in (75) equals

$$
\begin{aligned}
I & \equiv-2(2 \pi)^{-n} \int_{\mathbb{R}^{n}} \int_{\mathbb{R}^{n}} e^{-i x \cdot 1+i \varphi(x, \mu+i v)+i x} \cdot \eta(v-i \mu) \cdot \hat{A}(\eta) d \eta d x \\
& =2 i \int_{\mathbb{R}^{n}} e^{-i x \cdot l+i \varphi(x, \mu+i v)}(\mu+i v) \cdot A(x) d x .
\end{aligned}
$$

Comparing (76) with $\left(40^{\prime}\right)$, we see that

$$
(\mu+i v) \cdot \frac{\partial \varphi}{\partial x}=(\mu+i v) \cdot A(x),
$$

and hence, using the coordinates $\left(x_{1}, x_{2}, x^{\perp}\right)$ introduced before Proposition 2, we have

$$
I=2 \int_{\mathbb{R}^{n-2}} e^{-i \ell \cdot x^{\perp}}\left(\int_{\mathbb{R}^{2}}\left(\frac{\partial}{\partial x_{2}}+i \frac{\partial}{\partial x_{1}}\right) e^{i \varphi} d x_{1} d x_{2}\right) d x^{\perp} .
$$

We have

$$
\begin{gathered}
\int_{\mathbb{R}^{2}}\left(\frac{\partial}{\partial x_{2}}+i \frac{\partial}{\partial x_{1}}\right) e^{i \varphi} d x_{1} d x_{2}=\lim _{R \rightarrow \infty} \int_{x_{1}^{2}+x_{2}^{2} \leqq R^{2}}\left(\frac{\partial}{\partial x_{2}}+i \frac{\partial}{\partial x_{1}}\right) e^{i \varphi} d x_{1} d x_{2} \\
=\lim _{R \rightarrow \infty} \int_{0}^{2 \pi} e^{l \varphi\left(R \cos \theta, R \sin \left(x^{\perp}, \mu+l{ }^{\prime}\right)\right.}(\sin \theta+i \cos \theta) R \mathrm{~d} \theta
\end{gathered}
$$

by Green's theorem with $x_{1}=r \cos \theta$ and $x_{2}=r \sin \theta$. Returning to the expansion (52) for $\varphi$, we have

$$
\varphi=\frac{1}{2 \pi i} \frac{1}{x_{1}-i x_{2}} \int_{\mathbb{R}^{2}} A\left(y_{1} v+y_{2} \mu+x^{\perp}\right) \cdot(\mu+i v) d y_{1} d y_{2}+O\left(\left(x_{1}-i x_{2}\right)^{-2}\right) .
$$

Thus

$$
\begin{gathered}
\lim _{R \rightarrow \infty} \int_{0}^{2 \pi} e^{\imath \varphi\left(R \cos \theta, R \sin \theta, x^{\perp}, \mu+i v\right)}(\sin \theta+i \cos \theta) R \mathrm{~d} \theta \\
\quad=i \int_{\mathbb{R}^{2}} A\left(y_{1} v+y_{2} \mu+x^{\perp}\right) \cdot(\mu+i v) d y_{1} d y_{2}
\end{gathered}
$$

and

$$
\begin{aligned}
I & =2 i \int_{\mathbb{R}^{n-2}} e^{-i t \cdot x^{\perp}}\left(\int_{\mathbb{R}^{2}} A\left(y_{1} v+y_{2} \mu+x^{\perp}\right) \cdot(\mu+i v) d y_{1} d y_{2}\right) d x^{\perp} \\
& =2 i \hat{A}(l) \cdot(\mu+i v) .
\end{aligned}
$$

Since $\mu$ and $v$ are a general orthonormal pair perpendicular to $l$, we conclude that for all $l \in R^{n}, I$ determines $\hat{A}(l)-(\hat{A}(l) \cdot l) l /|l|^{2}$. In other words $I$ determines $A$ modulo the gradient of

$$
\rho(x)=(2 \pi)^{-n} \int_{\mathbb{R}^{n}} e^{l l \cdot x} i \hat{A}(l) \cdot l /|l|^{2} d l=-\Delta^{-1}(\nabla \cdot A),
$$

and hence $I$ determines curl A. 


\section{Recovering the Electric Potential}

To recover $V(x)$ we need to compute the next term in the asymptotic expansion of (69) which yielded (74) as the leading term. We have determined $A(x)$ modulo the gradient of a function of the form (78). Hence, we may assume that we know the scattering data for the problem with the $A(x)$ here and $q=$ $q^{\prime} \equiv A \cdot A-i \nabla \cdot A$, since the scattering data only depends on the magnetic field $B=\operatorname{curl} A$. This scattering data determines the Fourier transform of the solution $g_{0}$ of (53) with $f=f_{0} \equiv-\left(q^{\prime}+2(\zeta+z v) \cdot A(x)\right) \exp (i x \cdot \zeta)$ on the $\operatorname{set}(\xi, \zeta, z)=$ $(\xi(s), \zeta(s), z(s))$ given by (73). Among the operators in (69) only $T_{1}$ is changed when we replaced $g$ by $g_{0}$, and we denote the new operator by $T_{1,0}$. Thus, subtracting the representation (69) for $g_{0}$ from the representation (69) for $g$, we may assume that we know the Fourier transform on the curve $(\xi(s), \zeta(s), z(s))$ of

$$
\begin{gathered}
\left(I+(I+T)^{-1} C^{(-1)}\left(T_{1}+T_{2}+T_{3}\right)\right)^{-1}(I+T)^{-1} C^{(-1)}\left(f-f_{0}\right) \\
\left.-\left(I+(I+T)^{-1} C^{(-1)}\left(T_{1}+T_{2}+T_{3}\right)\right)^{-1}(I+T)^{-1} C^{(-1)}\left(T_{1}-T_{1,0}\right)\right) \\
\cdot\left(I+(I+T)^{-1} C^{(-1)}\left(T_{1,0}+T_{2}+T_{3}\right)\right)^{-1}(I+T)^{-1} C^{(-1)} f_{0} .
\end{gathered}
$$

Taking the limit in the Fourier transform of (79) at $(\xi(s), \zeta(s), z(s))$ as $s \rightarrow \infty$, we recover

$$
\begin{gathered}
\lim _{s \rightarrow \infty}(2 \pi)^{-n} \int_{\mathbb{R}^{n}} \int_{\mathbb{R}^{n}}-\hat{V}(\eta-\zeta(s)) e^{-l x \cdot(\xi(s)-\eta)+i \chi_{1}(\eta, z(s)) \varphi(x, \eta+z(s) v)} d \eta d x \\
-\lim _{s \rightarrow \infty} \mathscr{F}\left(C^{(-1)}\left(T_{1}-T_{1,0}\right) C^{(-1)} f_{0}\right)(\xi(s), \zeta(s), z(s)) \equiv J_{1}-J_{2} .
\end{gathered}
$$

By the same computation that derived (77) from (75), we have

$$
J_{1}=-\int_{\mathbb{R}^{n}} e^{-i x \cdot l+i \varphi(x, \mu+i v)} V(x) d x
$$

To compute $J_{2}$ we argue as follows. $T_{1}-T_{1,0}=V C L$, where $L$ multiplies the Fourier transform by $\left((\eta+z v) \cdot(\eta+z v)-k^{2}\right)^{-1}$. Since $[V, C]$ goes to zero and $C^{(-1)} C$ goes to the identity as $s \rightarrow \infty$, we can conclude that

$$
\begin{aligned}
& J_{2}=\lim _{s \rightarrow \infty}(2 \pi)^{-2 n} \int_{\mathbb{R}^{n}} \int_{\mathbb{R}^{n}} \int_{\mathbb{R}^{n}} \frac{\hat{V}(\xi(s)-\eta)}{(\eta+z(s) v) \cdot(\eta+z(s) v)-k^{2}} \\
& \times(-2(\zeta(s)+z(s) v) \cdot \hat{A}(\delta-\zeta(s))) \\
& \times e^{i x \cdot(\delta-\eta)+i \chi_{1}(\delta, z(s)) \varphi(x, \delta+z(s) v)} d \delta d x d \eta .
\end{aligned}
$$


Replacing $\delta$ by $\delta+\zeta(s)$ and $\eta$ by $\eta+\zeta(s)$, and arguing as before (recall $(\zeta(s)+$ $\left.z(s) v) \cdot(\zeta(s)+z(s) v)=k^{2}\right)$, we have

$$
\begin{aligned}
J_{2}= & (2 \pi)^{-2 n} \int_{\mathbb{R}^{n}} \int_{\mathbb{R}^{n}} \int_{\mathbb{R}^{n}} \frac{\hat{V}(l-\eta)}{2 \eta \cdot(\mu+i v)}(-2(\mu+i v) \cdot \hat{A}(\delta)) \\
& \times e^{i x \cdot(\delta-\eta)+i \varphi(x, \mu+i v)} d \delta d x d \eta \\
= & (2 \pi)^{-n} \int_{\mathbb{R}^{n}} \int_{\mathbb{R}^{n}}-\frac{\hat{V}(\ell-\eta)(\mu+i v) \cdot A(x) e^{-i x \cdot \eta+i \varphi\left(x, \mu+w^{\prime}\right)}}{(\mu+i v) \cdot \eta} d x d \eta .
\end{aligned}
$$

Proceeding as before with $x_{1}=x \cdot v$ and $x_{2}=x \cdot \mu$,

$$
\begin{aligned}
& \int_{\mathbb{R}^{n}} e^{-i x \cdot \eta+i \varphi\left(x, \mu+v^{\prime}\right)}(\mu+i v) \cdot A(x) d x \\
& \quad=\int_{\mathbb{R}^{n-2}} e^{-i x^{\perp} \cdot \eta^{\perp}} d x^{\perp} \int_{\mathbb{R}^{2}} e^{-i\left(x_{1} \eta_{1}+x_{2} \eta_{2}\right)}(-i)\left(\frac{\partial}{\partial x_{2}}+i \frac{\partial}{\partial x_{1}}\right)(\bar{c}(x, \mu+i v)-1) d x_{1} d x_{2},
\end{aligned}
$$

and by Green's theorem

$$
\begin{aligned}
& \int_{\mathbb{R}^{2}} e^{-i\left(x_{1} \eta_{1}+x_{2} \eta_{2}\right)}(-i)\left(\frac{\partial}{\partial x_{2}}+i \frac{\partial}{\partial x_{1}}\right)(\bar{c}-1) d x_{1} d x_{2} \\
& =\lim _{R \rightarrow \infty}\left[\int_{x_{1}^{2}+x_{2}^{2} \leqq R^{2}} e^{-i\left(x_{1} \eta_{2}+x_{2} \eta_{2}\right)}\left(\eta_{2}+i \eta_{1}\right)(\bar{c}-1) d x_{1} d x_{2}\right. \\
& \quad+\int_{0}^{2 \pi} e^{i R\left(\eta_{2} \cos \theta+\eta_{1} \sin \theta\right)} R(\sin \theta+i \cos \theta) \\
& \left.\quad \times\left(\bar{c}\left(R \cos \theta, R \sin \theta, x^{\perp}, \mu+i v\right)-1\right) d \theta\right] .
\end{aligned}
$$

Since

$$
\bar{c}\left(R \cos \theta, R \sin \theta, x^{\perp}, \mu+i v\right)-1=\frac{1}{2 \pi R} \cdot \frac{f\left(x^{\perp}\right)}{\cos \theta-i \sin \theta}+O\left(\frac{1}{R^{2}}\right),
$$

the second integral in the limit in (81) goes to zero as $R$ goes to infinity when $\left(\eta_{1}, \eta_{2}\right) \neq 0$. The first integral just goes to the Fourier transform of $\bar{c}-1$ in $\left(x_{1}, x_{2}\right)$ multiplied by $\left(\eta_{2}+i \eta_{1}\right)=(\mu+i v) \cdot \eta$. Thus

$$
J_{2}=-\int_{\mathbb{R}^{n}} e^{-i y \cdot l} V(y)\left(e^{i \varphi\left(y, \mu+i v^{\prime}\right)}-1\right) d y .
$$

Thus $J_{1}-J_{2}=-\int_{\mathbb{R}^{n}} e^{-l y} \cdot{ }^{l} V(y) d y$. Since $l$ is arbitrary, we have determined the Fourier transform of $V$ and the proof is complete. 


\section{References}

1. Agmon, S.: Spectral properties of Schrödinger operators and scattering theory. Annali di Pisa, Serie IV, 2, 151-218 (1975)

2. Eskin, G., Ralston, J.: The Inverse Backscattering Problem in Three Dimensions. Commun. Math. Phys. 124, 169-215 (1989)

3. Faddeev, L.D.: The inverse problem of quantum scattering II. J. Sov. Math. 5, 334-396 (1976)

4. Hörmander, L.: Uniqueness theorems for second order elliptic differential equations. Comm. in PDE 8, 21-64 (1983)

5. Nakamura, G., Sun, Z., Uhlmann, G.: Global Identifiability for an Inverse Problem for the Schrödinger Equation in a Magnetic Field. Preprint

6. Novikov, R.G., Khenkin, G.M.: The $\bar{\partial}$-equation in the multidimensional inverse scattering problem. Russ. Math. Surv. 42, 109-180 (1987)

7. Novikov, R.G.: The inverse scattering problem on a fixed energy level for the two-dimensional Schrödinger operator. J. Funct. Anal. 103, 409-463 (1992)

8. Novikov, R.G.: The inverse scattering problem at fixed energy for the three-dimensional Schrödinger equation with an exponentially decreasing potential. Commun. Math. Phys. 161, 569-595 (1994)

9. Sun, Z.: An inverse boundary value problem for Schrödinger operator with vector potentials. Trans of AMS 338 (2), 953-969 (1993)

Communicated by B. Simon 